\title{
The Star Formation Rate of Molecular Clouds
}

\author{
Paolo Padoan \\ University of Barcelona \\ Christoph Federrath \\ Monash University \\ Gilles Chabrier \\ Ecole Normale Suprieure de Lyon \\ Neal J. Evans II \\ The University of Texas at Austin \\ Doug Johnstone \\ University of Victoria \\ Jes K. Jørgensen \\ University of Copenhagen \\ Christopher F. McKee \\ University of California, Berkeley \\ Åke Nordlund \\ University of Copenhagen
}

\begin{abstract}
We review recent advances in the analytical and numerical modeling of the star formation rate in molecular clouds and discuss the available observational constraints. We focus on molecular clouds as the fundamental star formation sites, rather than on the larger-scale processes that form the clouds and set their properties. Molecular clouds are shaped into a complex filamentary structure by supersonic turbulence, with only a small fraction of the cloud mass channeled into collapsing protostars over a free-fall time of the system. In recent years, the physics of supersonic turbulence has been widely explored with computer simulations, leading to statistical models of this fragmentation process, and to the prediction of the star formation rate as a function of fundamental physical parameters of molecular clouds, such as the virial parameter, the rms Mach number, the compressive fraction of the turbulence driver, and the ratio of gas to magnetic pressure. Infrared space telescopes, as well as ground-based observatories have provided unprecedented probes of the filamentary structure of molecular clouds and the location of forming stars within them.
\end{abstract}

\section{INTRODUCTION}

Understanding and modeling the star formation rate (SFR) is a central goal of a theory of star formation. Cosmological simulations of galaxy formation demonstrate the impact of SFR models on galaxy evolution (Agertz et al., 2013), but they neither include star formation selfconsistently, nor its feedback mechanisms. They must rely instead on suitable subgrid-scale models to include the effects of star formation and feedback.

The SFR has been a fundamental problem in astrophysics since the 1970's, when it was shown that the gas depletion time in our Galaxy is much longer than any characteristic free-fall time of star forming gas (Zuckerman and Palmer, 1974; Williams and McKee, 1997). The same problem applies to individual MCs, where the gas depletion time is much longer than the fee-fall time as well (e.g. Evans et al., 2009). It has been argued that for local clouds (Krumholz and Tan, 2007), as well as for disk and starburst galaxies at low and high redshift (Krumholz et al., 2012a; Federrath, 2013b), the gas depletion time is always of the order of 100 free-fall times.

The first solution to the SFR problem was proposed under the assumption that MCs are supported against gravity 
by relatively strong magnetic fields, with star formation resulting from the contraction of otherwise subcritical cores by ambipolar drift (Shu et al., 1987). The relevant timescale for star formation would then be the ambipolar drift time, much longer than the free-fall time in MCs. By accounting for the effect of photoionization on the coupling of gas and magnetic field, McKee (1989) derived a model for the SFR that predicted gas depletion times consistent with the observations.

When it became possible to carry out relatively large three-dimensional simulations of magneto-hydrodynamic (MHD) turbulence in the 1990s, the focus of most SFR studies gradually moved from the role of magnetic fields to the role of supersonic turbulence, as described in a number of reviews (Scalo and Elmegreen, 2004; Elmegreen and Scalo, 2004; Mac Low and Klessen, 2004; BallesterosParedes et al., 2007; McKee and Ostriker, 2007). Padoan and Nordlund (1999) showed that the Zeeman-splitting measurements of the magnetic field in MCs were likely to indicate a mean magnetic field strength much weaker than previously assumed (see also Lunttila et al., 2008, 2009; Bertram et al., 2012). Based on a body of recent observational results, the review by McKee and Ostriker (2007) concludes that MCs are mostly supercritical instead of subcritical, in which case the ambipolar-drift time cannot be the solution to the SFR problem.

Current simulations of star formation by supersonic turbulence show a high sensitivity of the SFR to the virial parameter, defined as twice the ratio of turbulent kinetic energy to gravitational energy (Padoan and Nordlund, 2011; Federrath and Klessen, 2012; Padoan et al., 2012), and thus to the ratio between the free-fall time and the turbulence crossing time, as suggested by analytical models based on the turbulent fragmentation paradigm (Krumholz and McKee, 2005; Padoan and Nordlund, 2011; Hennebelle and Chabrier, 2011; Federrath and Klessen, 2012; Hennebelle and Chabrier, 2013). Interestingly, the models and simulations also show that the magnetic field is still needed to make the SFR as low as observed in MCs, even if the SFR is not controlled by ambipolar drift.

Most studies of the SFR published after Protostars and Planets V have focused on the role of turbulence, so this review revolves around supersonic turbulence as well. Besides the theoretical ideas and the numerical simulations, we discuss the observations that are used to constrain the SFR in MCs and have a potential to test the theory. Extragalactic observations can be used to constrain SFR models as well (Krumholz et al., 2012a; Federrath, 2013b). They present the advantage of a greater variety of star formation environments relative to local MCs, but they are not as detailed as studies of nearby clouds. Although the extragalactic field is rapidly evolving and very promising, the observational side of this review is limited to studies of local clouds, and we only briefly discuss the issue of relating the local studies to the extragalactic literature. For more information on this topic, we refer the reader to the recent review by Kennicutt and Evans (2012), and to the chapter by Dobbs et al. in this book.

We start by reviewing observational estimates of the SFR in MCs in Section 2, with a critical discussion of the methods and their uncertainties. We also contrast the methods based on the direct census of stellar populations in MCs, with indirect ones used to determine the SFR on larger scales in extragalactic studies.

We then review the theoretical models in Section 3, focusing on those that account for the turbulent nature of the interstellar medium, and discuss their differences, limitations, and potential for further development. The theory relies on statistical results from numerical studies of supersonic magnetohydrodynamic (MHD) turbulence, particularly on the probability distribution of gas density, whose dependence on fundamental physical parameters has been recently quantified in several studies. Besides the distribution of gas density, the key ingredient in the theory is the concept of a critical density for star formation. However, MCs are characterized by highly non-linear turbulent motions, producing shocks and filaments that are often described as fractal structures, casting doubt on such a threshold density concept. We discuss how the various models justify this approximation, and how the observations support the idea of a critical density.

Turbulence simulations have been used to directly derive the SFR, by introducing self-gravity and sink particles to trace gravitationally unstable and star-forming gas (e.g., Federrath et al., 2010b). Over the last two years, these simulations have been employed in vast parameter studies that can be used to test and constrain theoretical models. In Section 4 , we offer a critical view of numerical methods and experiments and we summarize the most important findings from the comparison between simulations and theories.

In Section 5 we compare both theory and simulations to the observational estimates of the SFR. We draw conclusions and outline future directions for both models and observations in Section 6.

\section{OBSERVATIONS}

Over the last decade infrared studies from ground-based telescopes, such as 2MASS (Kleinmann, 1992), and from space with the Spitzer Space Telescope (Werner et al., 2004) and Herschel Space Observatory (Pilbratt et al., 2010) have provided extended surveys of the populations of young stellar objects and their evolutionary stages, as well as their distributions within their parental clouds. Together with large-scale extinction and submillimeter continuum maps, which provide measurements of the cloud mass and column density, these observations allow direct estimates of the star formation rates and efficiencies in different cloud regions.

In writing this chapter, the authors realized that many misunderstandings were generated because of different conceptions of what a molecular cloud is. The obvious case is the "cloud in the computer" versus the "cloud imaged by observers", but even the latter is subject to definitional 
issues because of evolving sensitivity and observing techniques. We thus begin the next subsection with a discussion of techniques for measuring clouds and conclude with the evolving observational definition of a molecular cloud.

\subsection{Measuring Cloud Mass and Surface Density: Meth- ods and Uncertainties}

The distribution of mass in nearby molecular clouds has been determined primarily from extinction mapping in the visible and infrared, using Spitzer and 2MASS data, and from ground-based submillimeter surveys of dust continuum emission. These observations allow the surface densities and masses to be derived without assumptions about abundance and excitation of gas tracers, such as $\mathrm{CO}$ and its rarer isotopologues. The estimation of the total cloud mass does, however, rely on a consistency in the dust to gas ratio within and across clouds. These techniques have thus largely replaced maps of $\mathrm{CO}$ for nearby clouds, although $\mathrm{CO}$ is still required to uncover cloud kinematics. For more distant clouds, maps of $\mathrm{CO}$ and other species are still used, and we discuss the issues arising for cloud definition and properties in Section 2.3.

The advantages of the extinction mapping technique are twofold. First, the availability and sensitivity of large-area optical and infrared detectors allow large regions to be observed efficiently. Also, only the extinction properties of the intervening dust are required to convert from extinction to column density. The major disadvantages are the lack of resolution available, unless the infrared observations are extremely deep and the inability to measure very high column densities where the optical depth in the infrared becomes too large to see background stars.

Extinction maps from optical data have provided valuable measures of cloud extents, masses, and surface densities for modest extinctions (e.g., Cambrésy 1999; Dobashi et al. 2005), and the application to the near-infrared has extended these techniques to larger extinctions (e.g. Lombardi and Alves, 2001). The extinction of individual background stars are measured and converted to a column density of intervening gas and dust assuming appropriate properties for dust in molecular clouds. Studies of the extinction law in nearby clouds show that the dust is best represented by models with ratios of total to selective extinction, $R_{V}=5.5$ (Chapman et al. 2009; Ascenso et al. 2012, 2013). For the nearby clouds imaged with Spitzer, $2 \times 10^{4}$ to $1 \times 10^{5}$ background stars were identified (Evans et al., 2009); when added to the 2MASS data base, extinctions up to $A_{V}=40$ mag can be measured with spatial resolution of about $270^{\prime \prime}$ (Heiderman et al., 2010). The conversion from $A_{V}$ to mass surface density is

$$
\Sigma_{\text {gas }}\left(\mathrm{g} \mathrm{cm}^{-2}\right)=\mu m_{H}\left[1.086 C_{\text {ext }}(V)\right]^{-1} A_{V}
$$

where $\mu=1.37$. $C_{\text {ext }}(V)$ is the extinction per column density of $\mathrm{H}$ nucleons, $N(\mathrm{H})=N(\mathrm{HI})+2 N\left(\mathrm{H}_{2}\right)$ (Draine, 2003) (see on-line tables at http://www.astro. princeton.edu/ draine/dust/dustmix.html), Two different grain models are available for $R_{V}=5.5$. For normalized Case A grains, the newer models, $C_{\text {ext }}(V)=$ $6.715 \times 10^{-22} \mathrm{~cm}^{2}$, and $\Sigma_{g a s}=15 A_{V} \mathrm{M}_{\odot} \mathrm{pc}^{-2}$. For Case B grains, $C_{\text {ext }}(V)=4.88 \times 10^{-22} \mathrm{~cm}^{2}$, and $\Sigma_{\text {gas }}=$ $21 A_{V} \mathbf{M}_{\odot} \mathrm{pc}^{-2}$. The Case $\mathrm{B}$ grains match observations well (Ascenso et al., 2013), but have some theoretical issues (Draine, personal communication). Following Heiderman et al. (2010) we adopt the normalized Case A grain model, noting the possibility that all masses and surface densities are about $40 \%$ higher. Note that $C_{\text {ext }}=4.896 \times 10^{-22}$ $\mathrm{cm}^{2}$ (normalized Case A) or $5.129 \times 10^{-22} \mathrm{~cm}^{2}$ (Case B) for $R_{V}=3.1$, so use of the diffuse ISM conversions will result in higher estimates of $\Sigma_{\text {gas }}$. These values may apply to regions with $A_{V}<2 \mathrm{mag}$, but Ascenso et al. (2013) finds no clear change from $R_{V}=5.5$.

Ground-based submillimeter mapping of dust continuum emission provides an alternative, but it requires assumptions about both the dust emissivity properties at long wavelengths and the dust temperature distribution along the line of sight, usually taken to be constant. In addition, the ground-based submillimeter maps lose sensitivity to largescale structure. The resolution, however, can be significantly higher than for extinction mapping, $\sim 10$ 's of arcseconds rather than 100's, and the submillimeter emission remains optically thin even at extreme column densities. For a description of planned maps with SCUBA-2, see Johnstone et al. (2005) and Ward-Thompson et al. (2007b)

More recently Herschel has mapped a large fraction of the nearby molecular clouds from the far infrared through the submillimeter. The resulting spectral energy distributions add information about the mean dust temperature along the line of sight and thus yield more accurate column density maps of molecular clouds (e.g., Sadavoy et al. 2012; Kirk et al. 2013; Sadavoy et al. 2013; Schneider et al. 2013). The spatial resolution of Herschel lies between the standard extinction map scale and the ground-based submillimeter maps. We can expect a rich harvest of results once these surveys are fully integrated.

\subsection{The Mass and Surface Density of Clouds}

The three largest clouds in the c2d project were completely mapped at $1.1 \mathrm{~mm}$ using Bolocam on the CSO (Enoch et al. 2006; Young et al. 2006; Enoch et al. 2007). Additional maps were made at $850 \mu \mathrm{m}$ with SCUBA for Ophiuchus (Johnstone et al., 2004), Perseus (Hatchell and Fuller, 2008; Kirk et al., 2006), and Orion (Johnstone and Bally, 1999, 2006). Smaller clouds and regions were mapped at $350 \mu \mathrm{m}$ by $W u$ et al. (2007) providing higher resolution data at those wavelengths than was possible with Herschel. A uniform reprocessing of all SCUBA data provides a data base for many individual regions (di Francesco et al., 2006).

On larger scales, we now have surveys of the Galactic Plane at $\mathrm{mm} / \mathrm{smm}$ wavelengths, notably the Bolocam Galactic Plane Survey (BGPS) (Aguirre et al. 2011; 
Rosolowsky et al. 2010; Ginsburg et al. 2013) and the APEX Telescope Large Area Survey of the Galaxy (ATLASGAL) projects (Schuller et al. 2009; Contreras et al. 2013).

The primary product of these blind submillimeter surveys was a catalog of relatively dense structures. Although dust continuum emission is related to dust temperature and column density throughout the cloud, the loss of sensitivity to large scale emission for ground-based instruments results in an effective spatial filtering. This yields a particular sensitivity to small-scale column density enhancements and thus picks out structures with high volume density. For nearby clouds, mm sources correspond to cores, the sites of individual star formation (Enoch et al., 2006), whereas Galactic Plane surveys mostly pick up clumps, the sites of cluster formation (Dunham et al., 2011). This can also be seen in the fraction of cloud mass identified through the sub-mm mapping. In nearby clouds the sub-mm sources account for a few percent of the cloud mass (Johnstone et al., 2004; Kirk et al., 2006), while at larger distances the fraction of cloud mass observed appears to be much higher, reaching tens of percent, and the slope of the clump mass function flattens (Kerton et al., 2001; Muñoz et al., 2007).

For the nearby clouds, Spitzer data were used to distinguish protostellar cores from starless cores, almost all of which are probably prestellar (i.e. gravitationally bound), using the definitions of di Francesco et al. (2007) and WardThompson et al. (2007a). This distinction allowed a clarification of the properties of prestellar cores. Three results are particularly salient for this review.

First, the core mass function was consistent with a picture in which core masses map into stellar masses with a core-to-star efficiency of $\epsilon=0.25$ to 0.4 (Enoch et al. 2008; Sadavoy et al. 2010; Alves et al. 2007; André et al. 2010).

Second, the timescale for prestellar cores with mean densities above about $10^{4} \mathrm{~cm}^{-3}$ to evolve into protostellar cores is about 0.5 Myr (Enoch et al., 2008). Third, prestellar cores are strongly concentrated to regions of high extinction. For example, $75 \%$ of the cores lie above $A_{V}=8$, 15, and 23 mag in Perseus, Serpens, and Ophiuchus, respectively (Johnstone et al., 2004; Kirk et al., 2006; Enoch et al., 2007). These were some of the first quantitative estimates of the degree to which star formation is concentrated to a small area of the clouds characterized by high surface densities. In contrast, less than $20 \%$ of the area and $38 \%$ of the cloud mass lies above $A_{V}=8 \mathrm{mag}$ (Evans et al., submitted).

For 29 nearby clouds mapped by Spitzer, Evans et al. (submitted) have measured mean surface densities above the $A_{V}=2$ contour. These have a mean value of $\Sigma_{g a s}=$ $79 \pm 22 \mathrm{M}_{\odot} \mathrm{pc}^{-2}$. For maps of 12 nearby clouds that go down to $A_{V}=0.5$ mag with $0.1 \mathrm{pc}$ resolution, mean $A_{V}=1.6 \pm 0.7 \mathrm{mag}$ for star forming clouds and $A_{V}=$ $1.5 \pm 0.7 \mathrm{mag}$ if 5 non-star-forming clouds are included (Kainulainen et al., 2009). Clearly, the mean surface densities are heavily influenced by how low an extinction contour is included. These mean extinctions would correspond to 23 to $26 \mathrm{M}_{\odot} \mathrm{pc}^{-2}$ with the adopted extinction law, but other extinction laws could raise these values by about $40 \%$.

\subsection{Dust versus CO Observations}

The images of clouds from extinction or Herschel images of dust emission are qualitatively different from earlier ones. At low extinction levels, clouds appear wispy, windswept, and diffuse, more like cirrus clouds in our atmosphere than like cumulus clouds. The Herschel images tracing higher extinction regions, reveal a strong theme of filaments and strands with high contrast against the more diffuse cloud (see chapter by André et al.). These modern images of cloud properties must be contrasted with older images, which still dominate the mental images of molecular clouds for many astronomers. The very definition of molecular cloud depends on the technique used to measure it. For the nearby clouds, optical and near-infrared imaging define clouds to well below $A_{V}=1$ and it is only at $A_{V}<1$ that log-normal column density distributions become apparent, as discussed in later sections, while their SFR properties were generally measured down to only $A_{V}=2$, as discussed in the next section. In contrast, the maps of molecular clouds based on maps of CO probe down to a particular value of antenna temperature or integrated intensity, and the conversion to gas surface density depends on that limiting value.

The most detailed study comparing extinction and $\mathrm{CO}$ at low levels is that by Pineda et al. (2010), which combined deep extinction mapping with 0.14 pc resolution with extensive CO maps (Goldsmith et al., 2008). CO emission can be seen down to $A_{V} \sim 0.1 \mathrm{mag}$, but the conversion from $\mathrm{CO}$ emission to mass surface density rises rapidly as $A_{V}$ decreases below $1 \mathrm{mag}$. The mass in areas where ${ }^{13} \mathrm{CO}$ is not detected is roughly equal to the mass where it was detected. The net result is a mean surface density of 39 $\mathrm{M}_{\odot} \mathrm{pc}^{-2}$, based on the total cloud mass and area (Pineda et al., 2010).

For more distant clouds, or for clouds at low Galactic longitude and latitude, extinction and submillimeter maps cannot easily separate the cloud from the general Galactic field without additional kinematic data. The main tracer of clouds across the Galaxy has been maps of $\mathrm{CO}$ and isotopologues. One of the earliest and most influential studies of cloud properties was by Solomon et al. (1987), and this study remains the enduring image in the mental toolkit of many theorists and nearly all extragalactic astronomers. By defining clouds at a threshold of $3 \mathrm{~K}$ or higher in $T_{R}^{*}$ and assuming that the virial theorem applied, Solomon et al. (1987) derived a mean surface density for the inner Galaxy clouds of $\Sigma_{\text {gas }}=170 \mathrm{M}_{\odot} \mathrm{pc}^{-2}$, a value still quoted by many astronomers. When scaled to the newer distance to the Galactic Center, this value becomes $206 \mathrm{M}_{\odot} \mathrm{pc}^{-2}$ (Heyer et al., 2009). The Solomon et al. work was however based on severely undersampled maps, and the selection criteria favored "warm" clouds with active star formation. It has been superseded by better sampled and deeper 
maps of ${ }^{13} \mathrm{CO}$ by Heyer et al. (2009) and Roman-Duval et al. (2010). Heyer et al. (2009) found that the median surface density of inner Galaxy molecular clouds is 42 $\mathrm{M}_{\odot} \mathrm{pc}^{-2}$ within the extrapolated $1 \mathrm{~K}$ contour of $\mathrm{CO}$, but noted that abundance variations could raise the value to 80 $120 \mathrm{M}_{\odot} \mathrm{pc}^{-2}$. Roman-Duval et al. (2010) found a mean $\Sigma_{\text {gas }}=144 \mathrm{M}_{\odot} \mathrm{pc}^{-2}$ within the $4 \sigma$ contour of ${ }^{13} \mathrm{CO}$, which traced gas with a median $A_{V}>7$ mag, roughly. These values are thus measuring a fraction of the cloud with elevated surface densities, if those clouds are like the local clouds. Roman-Duval et al. (2010) find that $\Sigma_{\text {gas }}$ declines for Galactocentric radii beyond $6.6 \mathrm{kpc}$ to the lower values seen in nearby clouds. These values depend on assumptions about $\mathrm{CO}$ abundance and excitation. The definition and properties of clouds appear to depend on location in the Galaxy as well as on technique and sensitivity.

Extensive maps in molecular lines of the nearby clouds have also allowed studies of the dynamical properties of the clouds and locations of outflows (e.g., Ridge et al. 2006). These may also be used to compare to detailed simulations of line profiles from turbulent clouds.

\subsection{Measuring the SFR in MCs: Methods and Uncer- tainties}

Methods for measuring the SFRs in general have been reviewed and tabulated by Kennicutt and Evans (2012). The most direct measures of the SFRs in nearby molecular clouds are based on counting of young stellar objects (YSOs) and assigning a timescale to the observed objects. Recent surveys of molecular clouds with Spitzer provide a quite complete census of YSOs with infrared excesses (Evans et al., 2009; Dunham et al., 2013). There is however considerable uncertainty in the low luminosity range, where contamination by background objects becomes severe. Tradeoffs between completeness and reliability are inevitable. The YSO identifications in Evans et al. (2009) emphasized reliability; other methods have suggested $30 \%$ to $40 \%$ more YSOs (e.g., Kryukova et al. 2012; Hsieh and Lai 2013). Optical photometry and spectroscopy also provided valuable follow-up data for the later stages (e.g., Oliveira et al. 2009; Spezzi et al. 2010, 2008). Although we may expect updates as the Herschel surveys of the Gould Belt clouds are fully analyzed, initial results from the Orion clouds suggest that the percentage of sources identifiable only with Herschel data is about 5\% (Stutz et al., 2013).

The selection of YSOs by infrared excess means that older objects are not counted, but it has the advantage that the lifetime of infrared excess has been studied extensively by counting the fraction of stars with infrared excess in clusters of different ages. The half-life of infrared excess is determined to be $t_{\text {excess }}=2 \pm 1$ Myr (e.g., Mamajek 2009), with the main source of uncertainty being the choice of premain sequence (PMS) evolutionary tracks. All the measures of SFR $\left(\dot{M}_{*}\right)$ discussed later depend on the assumed halflife of infrared excesses. Increases in the ages of young clusters (see chapter by Soderblom et al.) will decrease the estimates proportionally. The third element needed is the mean mass of a YSO, for which we assume a fully sampled system IMF, with $\left\langle M_{\star}\right\rangle=0.5 \mathrm{M}_{\odot}$ (Chabrier, 2003). The nearby clouds are not forming very massive stars, so they are not sampling the full IMF; this effect would cause an overestimate of $\dot{M}_{*}$.

Putting these together,

$$
\left\langle\dot{M}_{*}\right\rangle=N(Y S O s)\left\langle M_{\star}\right\rangle / t_{\text {excess }} .
$$

This equation has been used to compute $\dot{M}_{*}$ for 20 nearby clouds (Evans et al., 2009; Heiderman et al., 2010), with the assumed $t_{\text {excess }}=2 \mathrm{Myr}$ being the major source of uncertainty (for a numerical study investigating the effect of varying $t_{\text {excess }}$, see Federrath and Klessen, 2012). From these measures of $\dot{M}_{*}$, one can compute surface densities of star formation rate, $\Sigma_{\mathrm{SFR}}$, efficiencies, defined by $\mathrm{SFE}=M(Y S O) /[M(Y S O)+M($ cloud $)]$ (see e.g., the definition in Federrath and Klessen, 2013), rates per mass (often referred to as efficiencies in extragalactic work), defined by $\dot{M}_{*} / M_{g a s}$, and their reciprocals, the depletion times, $t_{d e p}$. SFRs determined from star counting are the most reliable, but are available only for nearby clouds (e.g., Heiderman et al., 2010; Lada et al., 2010) reaching out to Orion (Megeath et al., 2012). However, even the Orion clouds are not fully representative of the regions of massive star formation in the inner galaxy or in other galaxies.

Other methods of measuring $\dot{M}_{*}$ are mostly taken from extragalactic studies, where star counting is impractical, and their application to the Galaxy is tricky. Among the methods listed in Kennicutt and Evans (2012), those that are not too sensitive to extinction, such as $24 \mu \mathrm{m}$ emission, total far-infrared emission, and thermal radio continuum, may be useful also in localized regions of the Galactic Plane. Vutisalchavakul and Evans (2013) tested a number of these and found that none worked well for nearby regions where star counts are available. They noted that all the extragalactic methods assume a well-sampled IMF and wellevolved cluster models, so their failure in nearby regions is not surprising. They did find consistency in estimated $\dot{M}_{*}$ between thermal radio and total far-infrared methods for regions with total far-infrared luminosity greater than $10^{4.5}$ $\mathrm{L}_{\odot}$. As long as the overall SFR in a region is dominated by massive, young clusters, the measures that are sensitive to only the massive stars work to within a factor of two (e.g., Chomiuk and Povich 2011).

In summary, methods using YSO counting are reasonably complete in nearby $(d<500$ pc) clouds, and SFRs, averaged over $2 \mathrm{Myr}$, are known to within a factor of about 2. For more distant clouds, where YSO counting is not practical, indirect measures can fail by orders of magnitude unless the region in question has a sufficiently well sampled IMF to satisfy the assumptions in models used to convert tracers into masses of stars.

\subsection{The Mass in Young Stars and the SFR in MCs}

The Spitzer/c2d survey summarized by Evans et al. 
(2009) mapped seven nearby star-forming regions, Perseus, Ophiuchus, Lupus I, III, and IV, Chamaeleon II, and Serpens. Additional data for JHK bands from the 2MASS project provided critical information for sorting YSOs from background stars and galaxies in Spitzer surveys (Harvey et al. 2007 for c2d and Gould Belt, Rebull et al. 2010 for Taurus, and Gutermuth et al. 2009 for Orion and nearby clusters).

Using the near-infrared extinction maps, these studies reveal a relatively small scatter in the star formation rate per unit area $\left(\Sigma_{\mathrm{SFR}}\right)$ of the c2d clouds. The values of $\Sigma_{\mathrm{SFR}}$ are in the range $0.65-3.2 \mathrm{M}_{\odot} \mathrm{Myr}^{-1} \mathrm{pc}^{-2}$, with an average of $1.6 \mathrm{M}_{\odot} \mathrm{Myr}^{-1} \mathrm{pc}^{-2}$. Efficiencies per $2 \mathrm{Myr}$, the characteristic timescale for young stellar objects that can be detected and characterized at these wavelengths, are generally small, $3 \%$ to $6 \%$. This is similar to the star formation efficiency (SFE) inferred from 2MASS studies of the Perseus, Orion $\mathrm{A}$ and B and MonR2 molecular clouds (Carpenter, 2000). In the smaller group L673, Tsitali et al. (2010) likewise find $\mathrm{SFE}=4.6 \%$. In contrast, Lada and Lada (2003) concluded that the SFE is larger for embedded clusters than for MCs as a whole. Typical values for embedded clusters are in the range $10-30 \%$, with lower values usually found for less evolved clusters.

These estimates largely differ because of the scales probed. For example, considering the larger scale Ophiuchus and Perseus clouds, Jørgensen et al. (2008) found that the SFE was strongly dependent on the column density and regions considered: the younger clusters such as L1688, NGC 1333 and IC 348 showed values of 10-15\%, contrasting with the SFE of a few \% found on larger scales (Jorgensen et al., 2008; Evans et al., 2009; Federrath and Klessen, 2013). Likewise, Maury et al. (2011) utilized the Herschel Space Observatory and IRAM 30 m observations to infer $\Sigma_{\mathrm{SFR}}=23 \mathrm{M}_{\odot} \mathrm{Myr}^{-1} \mathrm{pc}^{-2}$ for the Serpens South cluster (see also Gutermuth et al., 2008). Gutermuth et al. noted that the YSO surface density of the Serpens South cluster was $>430 \mathrm{pc}^{-2}$ within a circular region with a $0.2 \mathrm{pc}$ radius, corresponding to a higher $\Sigma_{\mathrm{SFR}} \approx 700 \mathrm{M}_{\odot} \mathrm{Myr}^{-1} \mathrm{pc}^{-2}-$ again likely reflecting the density of material there. Zooming-in on the material just associated with dense cores, the gas+dust mass becomes comparable to the mass of the young stellar objects - an indication that the efficiency of forming stars is high once material is in sufficiently dense cores (Enoch et al., 2008; Jørgensen et al., 2008).

In particular, Federrath and Klessen (2013) measured the SFE in 29 clouds and cloud regions with a new technique based on the column-density power spectrum. They find that the SFE increases from effectively zero in largescale HI clouds and non-star-forming clouds to typical star-forming molecular clouds $(\mathrm{SFE}=1 \%-10 \%)$ to dense cores (SFE > 10\%), where the SFE must eventually approach the core-to-star efficiency, $\epsilon \approx 0.3-0.7$. Undoubtedly, the SFE is not constant over time within a cloud. The observed SFE must depend both on the properties of a cloud and on its the evolutionary state. The above qualitative SFE sequence encapsulates both of these effects.

In systematic comparisons between clouds or substructures of similar scales, differences are also seen: Lada et al. (2010) combined the data for a sample of MCs and found a strong (linear) correlation between the SFR and the mass of the cloud at extinctions above $A_{K} \approx 0.8 \mathrm{mag}\left(A_{V} \approx 7.5\right.$ mag). Heiderman et al. (2010) and Gutermuth et al. (2011) examined the relation between the SFR and local gas surface density in subregions of clouds and likewise found relations between the two: Gutermuth et al. (2011) found that $\Sigma_{\text {SFR }} \propto \Sigma_{\text {gas }}{ }^{2}$, while Heiderman et al. (2010) found a relation with a steeper increase (faster than squared) at low surface densities and a less steep increase (linear) at higher surface densities. One difference between these studies was the inclusion of regions forming more massive stars: while the study by Gutermuth et al. includes SFRs based on direct counts for a number of clusters forming massive stars, the Heiderman et al. study is based on the far-infrared luminosities and $\mathrm{HCN}$ column densities for a sample of distant, more active, star-forming regions, which may underestimate the true SFRs (Heiderman et al., 2010; Vutisalchavakul and Evans, 2013).

Another result of these studies is the presence of a star formation threshold in surface density: Heiderman et al. (2010) and Lada et al. (2010) find evidence for a characteristic extinction threshold of $A_{V} \approx 7$ to 8 mag separating regions forming the great majority of stars from those where star formation is rare. Similar thresholds have previously been suggested, e.g., on the basis of mapping of $\mathrm{C}^{18} \mathrm{O}$ emission (Onishi et al., 1998) and (sub)millimeter continuum maps (e.g., Johnstone et al., 2004; Kirk et al., 2006; Enoch et al., 2007) of a number of the nearby star-forming clouds. With our adopted conversion (see $\$ 2.1$ ), the extinction threshold translates to gas surface density of about $120 \pm 20 M_{\odot} \mathrm{pc}^{-2}$, but other conversions would translate to about $160 M_{\odot} \mathrm{pc}^{-2}$ (see, e.g., Lada et al. 2013).

All these methods measure the SFR for relatively nearby star-forming regions where individual young stellar objects can be observed and characterized. An alternative on larger scales is to measure the SFR using the free-free emission, for example from the Wilkinson Microwave Anisotropy Probe (WMAP). The free-free emission is expected to be powered by the presence of massive stars through the creation of HII regions. Using this method and by comparing to the GMC masses from CO line maps, Murray (2011) find varying star formation efficiencies ranging from $0.2 \%$ to $20 \%$ with an average of $8 \%$. However, as the author notes, the sample is biased toward the most luminous sources of free-free emission, potentially selecting the most actively star-forming regions in our Galaxy.

Observationally, the SFR scales with the cloud mass and, with a much reduced scatter, on the mass of "dense" gas (Lada et al. 2010, 2012). Figure 1 shows the log of the $\mathrm{SFR} / \mathrm{mass}$ versus the mass, using either the mass of dense gas $\left(A_{V}>8 \mathrm{mag}\right)$, or the full cloud mass, down to $A_{V} \sim 2$ mag. The large scatter in the star formation rate per unit total gas mass and the much smaller scatter per unit mass 

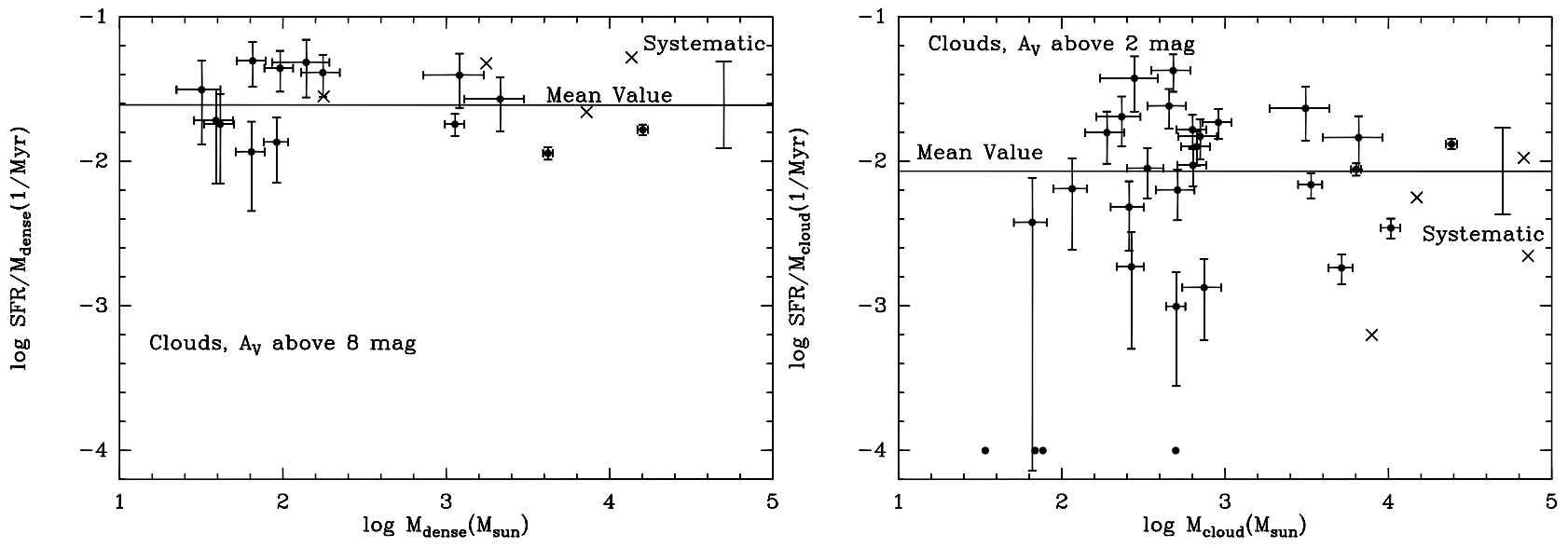

Fig. 1.- The log of the SFR per mass of dense gas versus the log of the mass of dense gas (left panel) and the log of the SFR per total cloud mass versus the log of the total cloud mass (right panel). The filled circles are based on the c2d and Gould Belt clouds (Evans et al. submitted), while the crosses represent Orion A, Orion B, Taurus, and the Pipe, taken from Lada et al. (2010). While there are some differences in identification and selection of YSOs, they are small. On the left panel, the extinction contour defining the dense gas is $A_{V}=8 \mathrm{mag}$ for clouds taken from Evans et al. and $A_{K}=0.8 \mathrm{mag}$ for those taken from Lada et al. (2010). On the right panel, the extinction contour defining the cloud is usually $A_{V}=2$ mag for clouds taken from Evans et al. and $A_{K}=0.1$ mag for those taken from Lada et al. (2010). Uncertainties on observables, including cloud distance, have been propagated for the $\mathrm{c} 2 \mathrm{~d}$ and Gould Belt clouds; the requisite information is not available for the "Lada" clouds. The four points plotted at -4 on the y axis are clouds with no observed star formation. The horizontal lines show the mean values for the $\mathrm{c} 2 \mathrm{~d}$ and Gould Belt clouds and the error bars represent the likely systematic uncertainties, dominated by those in the SFR.

of dense gas are clearly illustrated. The average values for the c2d plus Gould Belt clouds are as follows (Evans et al. submitted): $t_{d e p}=201 \pm 240 \mathrm{Myr}$ for clouds, $47 \pm 24 \mathrm{Myr}$ for dense gas; $t_{\mathrm{ff}}=1.47 \pm 0.58 \mathrm{Myr}$ for clouds, $0.71 \pm 0.38$ Myr for dense gas; $\mathrm{SFR}_{\mathrm{ff}} \equiv t_{\mathrm{ff}} / t_{\text {dep }}=0.018 \pm 0.014$ for clouds, $0.018 \pm 0.008$ for dense gas.

Removing this first-order dependence by looking at variations in SFR per mass of either cloud or dense gas versus other, non-dimensional parameters is probably the best way forward. In doing so, we need to be clear about the entity in which we are comparing the SFRs. It could be for the whole cloud, defined by either extinction or $\mathrm{CO}$ emission, most of which lies well below the dense gas threshold, or it could refer only to the denser gas, for which the dispersion is much smaller. The latter might best be thought of as the star forming portion of the cloud, which is often called a clump (Williams et al., 2000).

\subsection{Comparison with Extragalactic Results}

As many surveys of the Milky Way are becoming available, it is increasingly interesting to put our Galaxy in the context of galactic star formation studies. For other galaxies, the standard way to look at star formation is via the Kennicutt-Schmidt (KS) relation (Kennicutt, 1998b,a). Generalizing earlier suggestions (Schmidt, 1959, 1963), involving a relation between star formation and gas volume densities, Kennicutt (1998b) found a relation between more readily measured surface densities of the form:

$$
\Sigma_{\mathrm{SFR}} \propto \Sigma_{\text {gas }}{ }^{N}
$$

with $N \approx 1.4$ providing a good fit over many orders of magnitude for averages over whole galaxies.

Studies of resolved star formation over the face of nearby galaxies reveal a more complex picture with a threshold around $\Sigma_{\text {gas }}=10 \mathrm{M}_{\odot} \mathrm{pc}^{-2}$, roughly coincident with the transition from atomic to molecular-gas dominated interstellar media, and a linear dependence on the surface density of molecular gas, $\Sigma_{m o l}$, above that threshold (Bigiel et al., 2010). Another transition around $\Sigma_{m o l}=100$ $\mathrm{M}_{\odot} \mathrm{pc}^{-2}$, where the transition from normal to starburst galaxies is found, has been suggested (Genzel et al., 2010; Daddi et al., 2010), but this transition depends on interpretation of the $\mathrm{CO}$ observations, particularly the uncertainty in the extrapolation of the total column density. The existence of this second transition is consistent with relations between star formation and dense gas measured by $\mathrm{HCN}$ emission found earlier by Gao and Solomon (2004) and extended to clumps forming massive stars in the Milky Way by Wu et al. (2005). Together with the evidence for a sharp increase in SFR above about $\Sigma_{\text {gas }}=120 \mathrm{M}_{\odot} \mathrm{pc}^{-2}$ in local clouds, these results led Kennicutt and Evans (2012) to suggest a second transition to a more rapid form of star formation around $\Sigma_{m o l} \approx 100-300 \mathrm{M}_{\odot} \mathrm{pc}^{-2}$, depending on the physical conditions.

When the nearby clouds are plotted on the KS relation, all but the least active lie well above the relation for whole galaxies or the linear relation found in nearby galaxies (Evans et al., 2009). They are more consistent with the relation for starburst galaxies and the dense gas relations noted above (Heiderman et al., 2010). Federrath and 
Klessen (2012), Krumholz et al. (2012a), and Federrath (2013b) provide suggestions to resolve this discrepancy between extra-galactic and Milky Way SFRs.

\section{THEORY}

The complexity of turbulence, especially in the supersonic regime and in the case of magnetized and selfgravitating gas, precludes the development of fully analytical theories of the dynamics of MCs. However, the non-linear chaotic behavior of turbulent flows results in a macroscopic order that can be described with universal statistics. These statistics are flow properties that only depend on fundamental non-dimensional parameters expressing the relative importance of various terms in the underlying equations, such as the Reynolds and Mach numbers. Once established, scaling laws and probability density functions (PDFs) of turbulent flow variables can be applied to any problem involving turbulent flows with known parameters. Furthermore, because of the chaotic nature of turbulent flows, their steady-state statistics cannot even depend on the initial conditions. Because of this universality of turbulence, the statistical approach to the dynamics of MCs is a powerful tool to model the MC fragmentation induced by supersonic turbulence (with the obvious caveat that each $\mathrm{MC}$ is an individual realization of a turbulent flow, with specific local forces and possibly significant deviations from isotropy and steady state).

For many astrophysical problems involving supersonic turbulence, the turbulent fragmentation, that is the creation of a highly nonlinear density field by shocks, is the most important effect of the turbulence. One can then tackle those problems by focusing on the end result, the statistics of density fluctuations, largely ignoring the underlying dynamics. The PDF of gas density in supersonic turbulence is then the statistics of choice. This is especially true in modeling star formation, because the density enhancements, under characteristic MC conditions, are so large that the turbulence alone can seed the gravitational collapse of compressed regions directly into the very non-linear regime. In other words, much of the fragmentation process is directly controlled by the turbulence (the non-linear coupling of the velocity field on different scales).

These considerations explain the choice of part of the numerical community, starting in the 1990's, to focus the research on idealized numerical experiments of supersonic turbulence, using periodic boundary conditions, isothermal equation of state, random driving forces, and, notably, no self-gravity (e.g. Mac Low et al., 1998; Padoan and Nordlund, 1999; Mac Low et al., 1998; Padoan et al., 2004; Kritsuk et al., 2007; Federrath et al., 2010a). These idealized simulations have stimulated important theoretical advances and better understanding of supersonic turbulence (e.g. Falkovich et al., 2010; Aluie, 2011; Galtier and Banerjee, 2011; Kritsuk et al., 2013; Aluie, 2013). The setup of these experiments (though with the inclusion of selfgravity and sink particles) are described in Section 4. Here we briefly summarize the most important numerical results regarding the gas density PDF (Section 3.1). We then introduce the concept of a critical density for star formation (Section 3.2), and discuss its application to the prediction of the SFR (Section 3.3).

\subsection{The PDF of Gas Density in Supersonic Turbulence}

The gas density PDF was first found to be consistent with a log-normal function in three-dimensional simulations of compressible homogeneous shear flows by Blaisdell et al. (1993), in two-dimensional simulations of supersonic turbulence by Vazquez-Semadeni (1994), and in three-dimensional simulations of supersonic turbulence by Padoan et al. (1997b). The log-normal function it is fully determined by its first and second order moments, the mean and the standard deviation, as it is defined by a trivial transformation of the Gaussian distribution: if the PDF of $\widetilde{\rho}=\rho / \rho_{0}$ is log-normal ( $\rho_{0}$ is the mean density), then the PDF of $s=\ln (\widetilde{\rho})$ is Gaussian:

$$
p(s) d s=\frac{1}{\left(2 \pi \sigma_{s}^{2}\right)^{1 / 2}} \exp \left[-\frac{\left(s-s_{0}\right)^{2}}{2 \sigma_{s}^{2}}\right] d s
$$

where $s_{0}=-\sigma_{s}^{2} / 2$. Padoan et al. (1997a) and Nordlund and Padoan (1999) found that the standard deviation of the density scales linearly with the Mach number, $\sigma_{\widetilde{\rho}}=b \mathcal{M}_{\mathrm{s}}$, where $\mathcal{M}_{\mathrm{s}} \equiv \sigma_{\mathrm{v}} / c_{\mathrm{s}}$, and $b \approx 1 / 2$. This relation and the value of $b$ can be derived from a simple model based on a single shock (Padoan and Nordlund, 2011). In terms of the logarithmic density, $s$, this is equivalent to,

$$
\sigma_{s}^{2}=\ln \left[1+b^{2} \mathcal{M}_{\mathrm{s}}^{2}\right]
$$

The log-normal nature of the PDF is valid only in the case of an isothermal equation of state (at low densities, time-averaging may be necessary to cancel out deviations due to large-scale expansions and retrieve the full lognormal shape). Strong deviations from the log-normal PDF can be found with a (non-isothermal) polytropic equation of state (Passot and Vázquez-Semadeni, 1998; Scalo et al., 1998; Nordlund and Padoan, 1999). However, Glover et al. (2010) and Micic et al. (2012) find nearly log-normal PDFs when a detailed chemical network including all relevant heating and cooling processes is taken into account. Further simulations tested the validity of eq. (5) and derived more precise values of $b$ (e.g. Ostriker et al., 1999; Klessen, 2000; Glover and Mac Low, 2007; Kritsuk et al., 2007; Federrath et al., 2008a; Schmidt et al., 2009; Federrath et al., 2010a; Price et al., 2011; Konstandin et al., 2012; Federrath, 2013a). Other numerical studies considered the effect of magnetic fields (e.g. Ostriker et al., 2001; Lemaster and Stone, 2008; Padoan and Nordlund, 2011; Molina et al., 2012), and the effect of gravity (e.g. Klessen, 2000; Federrath et al., 2008b; Collins et al., 2011; Kritsuk et al., 2011b; Cho and Kim, 2011; Collins et al., 2012; Federrath and Klessen, 2013; Kainulainen et al., 2013a). 
The value of $b$ depends on the ratio of compressional to total power in the driving force, with $b \approx 1$ for purely compressive driving, and $b \approx 1 / 3$ for purely solenoidal driving (Federrath et al., 2008a, 2010a). It has also been found that the PDF can be very roughly described by a log-normal also in the case of magnetized turbulence, with a simple modification of eq. (5) derived by Padoan and Nordlund (2011) and Molina et al. (2012),

$$
\sigma_{s}^{2}=\ln \left[1+b^{2} \mathcal{M}_{\mathrm{s}}^{2} \beta /(\beta+1)\right],
$$

where $\beta$ is the ratio of gas to magnetic pressures, $\beta=$ $8 \pi \rho c_{\mathrm{S}}^{2} / B^{2}=2 c_{\mathrm{S}}^{2} / v_{\mathrm{A}}^{2}=2 \mathcal{M}_{\mathrm{A}}^{2} / \mathcal{M}_{\mathrm{s}}^{2}$ for isothermal gas, $v_{\mathrm{A}} \equiv B / \sqrt{4 \pi \rho}$ is the Alfvén velocity, and $\mathcal{M}_{\mathrm{A}} \equiv \sigma_{\mathrm{v}} / v_{\mathrm{A}}$ is the Alfvénic Mach number. This relation simplifies to the non-magnetized case (eq. (5)) as $\beta \rightarrow \infty$. The derivation accounts for magnetic pressure, but not magnetic tension. In other words, it neglects the anisotropy of MHD turbulence that tends to align velocity and magnetic field. The anisotropy becomes important for a large magnetic field strength, and the relation (6) is known to break down for trans- or sub-Alfvénic turbulence, requiring a more sophisticated model.

It has been found that self-gravity does not affect the density PDF at low and intermediate densities, and only causes the appearance of a power-law tail at large densities, reflecting the density profile of collapsing regions (Klessen, 2000; Dib and Burkert, 2005). Kritsuk et al. (2011b) have used a very deep AMR simulation covering a range of scales from 5 pc to 2 AU (Padoan et al., 2005), where individual collapsing cores are well resolved, and the density PDF develops a power law covering approximately 10 orders of magnitude in probability. By comparing the density profiles of the collapsing cores with the power-law high-density tail of the density PDF, they demonstrated that the PDF tail is consistent with being the result of an ensemble of collapsing regions. They point out that a spherically symmetric configuration with a power-law density profile, $\rho=\rho_{0}\left(r / r_{0}\right)^{-n}$ has a power-law density PDF with slope $m$ given by $m=-3 / n$, and a projected density PDF with slope $p$ given by $p=-2 /(n-1)$ (see also Tassis et al. (2010) and Girichidis et al. (2013)). They find that in their simulation the power-law exponent decreases over time, and may be nearly stationary at the end of the simulation, when it reaches a value $m=-1.67$ for the density PDF, and $p=-2.5$ for the projected density PDF. Both values give $n=-1.8$, consistent with similarity solutions of the collapse of isothermal spheres (Whitworth and Summers, 1985). Collins et al. (2011) find a very similar slope, $m=-1.64$, also in the case of magnetized turbulence, and Federrath and Klessen (2013) show that this result does not depend on the Mach number or on the ratio of compressive to total power, consistent with being primarily the effect of self-gravity in collapsing regions. The slope has a slight dependence on the turbulence Alfvénic Mach number, as the absolute value of $m$ increases with increasing magnetic field strength (Collins et al., 2012).

\subsection{The Critical Density for Star Formation}

Current theories assume that molecular clouds are supersonically turbulent and that pre-stellar cores arise as gravitationally unstable density fluctuations in the turbulent flow. As explained in Section 3.3, they derive the SFR as the mass fraction above an effective critical density for star formation, $\rho_{\text {crit }}$, which can be computed by assuming that the density PDF is log-normal, as discussed in Section 3.1. The derivation of such a critical density is therefore a crucial step in current SFR models. Federrath and Klessen (2012) have summarized the various treatments of the critical density in recent theories of the SFR by Krumholz and McKee (2005, KM), Padoan and Nordlund (2011, PN), and Hennebelle and Chabrier (2011, 2013, HC). Table 1 summarizes the main differences in those theories, in particular the different choice of density threshold.

One of the fundamental dimensionless parameters in the theories is the virial parameter, given by the ratio of turbulent and gravitational energies. Because of the difficulty of estimating the virial parameter in non-idealized systems, we distinguish between the theoretical virial parameter, $\alpha_{\mathrm{vir}, \mathrm{T}}=2 E_{\mathrm{kin}} / E_{\text {grav }}$, and the observational one:

$$
\alpha_{\mathrm{vir}, \mathrm{O}}=5 \sigma_{\mathrm{v}, 1 \mathrm{D}}^{2} R / G M,
$$

where $E_{\text {kin }}$ and $\sigma_{\mathrm{v}, 1 \mathrm{D}}$ include thermal effects. The two virial parameters are equal for an isothermal, spherical cloud of constant density (Bertoldi and McKee, 1992; Federrath and Klessen, 2012); for centrally concentrated clouds, $\alpha_{\text {vir, O }}>\alpha_{\text {vir, T. Federrath and Klessen (2012) }}$ find that the fractal structure (e.g., Scalo, 1990; Federrath et al., 2009) of the clouds and the assumed boundary conditions (particularly periodic vs. non-periodic) can lead to order-of-magnitude differences between $\alpha_{\text {vir, o }}$ and $\alpha_{\text {vir, T }}$. In the absence of a magnetic field, isolated clouds with $\alpha_{\text {vir, T }}<1$ cannot be in equilibrium and will collapse. Including the effects of surface pressure, the critical value of $\alpha_{\text {vir, T }}$ for collapse is somewhat greater than 1 (Bertoldi and McKee, 1992). The corresponding critical value for $\alpha_{\text {vir, o depends on the density distribution; for example, for }}$ a thermally supported Bonnor-Ebert sphere, it is 2.054. In the following, we refer to the virial parameter on the cloud diameter scale $L=2 R$ as $\alpha_{\mathrm{cl}}$.

KM assumed that low-mass pre-stellar cores are thermally supported, so that in a turbulent medium the Jeans length of a critical core is proportional to the 1D sonic length, $\lambda_{\mathrm{J} \text {, crit }}=\ell_{\mathrm{s}, 1 \mathrm{D}} / \phi_{x}$, where $\phi_{x}$ is a numerical factor that was assumed to be of order unity. They derived the sonic length using a linewidth-size relation of the form $\sigma_{\mathrm{v}, 1 \mathrm{D}} \propto r^{q}$. In their numerical evaluation, they adopted $q \simeq 1 / 2$, consistent with both simulations (e.g. Padoan, 1995; Kritsuk et al., 2007; Federrath et al., 2010a; Federrath, 2013a) and observations (e.g. Ossenkopf and Mac Low, 2002; Heyer and Brunt, 2004; Padoan et al., 2006, 2009; Roman-Duval et al., 2011). As a result, they obtained

$$
\rho_{\mathrm{crit}, \mathrm{KM}}=\left(\pi^{2} \phi_{x}^{2} / 45\right) \alpha_{\mathrm{cl}} \mathcal{M}_{\mathrm{s}, \mathrm{cl}}^{2} \rho_{\mathrm{cl}},
$$


where $\alpha_{\mathrm{cl}}$ is the observational virial parameter for the cloud, $\rho_{\mathrm{cl}}$ is the mean density of the cloud, and $\mathcal{M}_{\mathrm{s}, \mathrm{cl}}$, like all Mach numbers in this paper, is the 3D Mach number. They estimated the factor $\phi_{x}$ to be 1.12 from comparison with the simulations of Vázquez-Semadeni et al. (2003). If the sonic length is defined based on the 3D velocity dispersion, then the coefficient in Equation (8) changes from $\left(\pi^{2} \phi_{x}^{2} / 45\right)$ to $\left(\pi^{2} \phi_{x}^{2} / 5\right)$ (Federrath and Klessen, 2012). The KM result for $\rho_{\text {crit }}$ is a generalization of the result of Padoan (1995) that $\rho_{\text {core, crit }} \simeq \mathcal{M}_{\mathrm{s}, \mathrm{cl}}^{2} \rho_{\mathrm{cl}}$.

In $\mathrm{PN}$, the critical density was obtained by requiring that the diameter of a critical Bonnor-Ebert sphere be equal to the characteristic thickness of shocked layers (identified as the size of prestellar cores), inferred from isothermal shock conditions (MHD conditions when the effect of the magnetic field is included):

$$
\rho_{\text {crit }, \mathrm{PN}} \simeq 0.067 \theta_{\mathrm{int}}^{-2} \alpha_{\mathrm{cl}} \mathcal{M}_{\mathrm{s}, \mathrm{cl}}^{2} \rho_{\mathrm{cl}},
$$

where $\theta_{\text {int }}=$ integral-scale radius/cloud diameter $\approx 0.35$. PN did not assume a velocity-size relation, so their result does not depend on a specific value of $q$. However, for a reasonable value of $q=1 / 2$, the critical density in KM agrees with that in PN to within a factor 2 .

The virial parameter can also be expressed as $\alpha_{\mathrm{cl}} \propto$ $\sigma_{\mathrm{v}, \mathrm{cl}}^{2} / \rho_{\mathrm{cl}} R_{\mathrm{cl}}^{2}$. Thus, if the exponent of the line width-size relation is $q=1 / 2$, the critical density from equations (8) and (9) becomes independent of cloud density, virial parameter and Mach number:

$$
n_{\mathrm{H}, \mathrm{crit}} \approx(5-10) \times 10^{4} \mathrm{~cm}^{-3}\left(T_{\mathrm{cl}} / 10 \mathrm{~K}\right)^{-1},
$$

assuming $\sigma_{\mathrm{v}, 1 \mathrm{D}}=0.72 \mathrm{~km} \mathrm{~s}^{-1} \mathrm{pc}^{-1 / 2}(R / 1 \mathrm{pc})^{1 / 2}(M c$ Kee and Ostriker, 2007). This previously overlooked result suggests a possible theoretical explanation for the nearly constant threshold density for star formation suggested by observations (see Sections 2 and 5.3). However, it must be kept in mind that $n_{\mathrm{H} \text {, crit }}$ varies as the square of the coefficient in the linewidth-size relation, so regions with different linewidth-size relations should have different critical densities. It should also be noted that magnetic fields tend to reduce the critical density by an uncertain amount (see below), and that the $\mathrm{HC}$ formulation of the critical density does not yield a constant value.

If the core is turbulent, McKee et al. (2014, in preparation) show that the critical density can be expressed as

$$
\rho_{\text {core }, \text { crit }} \simeq\left(\frac{\sigma_{\mathrm{v}, \mathrm{cl}}}{\sigma_{\mathrm{v}, \mathrm{core}}}\right)^{2} \frac{\alpha_{\mathrm{cl}}}{\alpha_{\mathrm{crit}}} \rho_{\mathrm{cl}} .
$$

The numerical factor $\alpha_{\text {crit }}=\mathcal{O}(1)$ must be determined from theory or simulation, but the virial parameter of the cloud, $\alpha_{\mathrm{cl}}$, can be determined from observations. Cores that are turbulent can be subject to further fragmentation, but this is suppressed by protostellar feedback (e.g., Myers et al., 2013). For cores that are primarily thermally supported $\left(\sigma_{\mathrm{v}, 1 \mathrm{D}, \text { core }} \simeq c_{\mathrm{S}}\right)$, this expression has the same form as the KM and PN results above; if the core is turbulent, the critical density is reduced.
$\mathrm{HC}$ include the effects of turbulence in pre-stellar cores, but they argue that their model does not have a critical density for star formation. That is true of their derivation of the stellar IMF, where cores of different densities are allowed to collapse. For example, large turbulent cores with density lower than that of thermally supported cores are at the origin of massive stars, as in McKee and Tan (2003). However, when integrating the mass spectrum of gravitationally unstable density fluctuations to derive the SFR, HC face the problem of choosing the largest mass cutoff in the integral, corresponding to the most massive star, which introduces an effective critical density. They chose to extend the integral up to a fraction, $y_{\text {cut }}$, of the cloud size, $L_{\text {cl }}$. Their critical density is thus the density such that the Jeans length (including turbulent support) is equal to $y_{\mathrm{cut}} L_{\mathrm{cl}}$ :

$$
\rho_{\mathrm{crit}, \mathrm{HC}}=\left[\left(\frac{\pi^{2}}{5}\right) y_{\mathrm{cut}}^{-2} \mathcal{M}_{\mathrm{s}, \mathrm{cl}}^{-2}+\left(\frac{\pi^{2}}{15}\right) y_{\mathrm{cut}}^{-1}\right] \alpha_{\mathrm{cl}} \rho_{\mathrm{cl}},
$$

where the first term is the contribution from thermal support, and the second term the contribution from turbulent support. The second term is larger than the first one if $\mathcal{M}_{\mathrm{s}, \mathrm{cl}}>\left(3 / y_{\text {cut }}\right)^{1 / 2}$, and can be obtained from equation (11) by noting that, for $q=\frac{1}{2}$, one has $\mathcal{M}_{\mathrm{s}, \text { core, cut }}^{2} / \mathcal{M}_{\mathrm{s}, \mathrm{cl}}^{2}=R_{\text {core, cut }} / R_{\mathrm{cl}}=y_{\text {cut }}$ for the maximum core mass; the second term of equation (12) then follows by taking $\alpha_{\text {core }}=15 / \pi^{2}$, similar to KM. HC assume that $y_{\text {cut }}$ is a constant, independent of the properties of the cloud; the physical justification for this has yet to be provided. In summary, the critical density for KM and $\mathrm{PN}$ is defined for thermally supported cores, whereas that for $\mathrm{HC}$ is for the turbulent core corresponding to the most massive star.

Besides affecting the density PDF in a complex way (see Section 3.1), magnetic fields can alter the critical density. Equation (11) remains valid, but the values of the virial parameters change. McKee and Tan (2003) estimated that $\alpha_{\text {vir, O }}$ is reduced by a factor $\left(1.3+1.5 / \mathcal{M}_{\mathrm{A}}^{2}\right)$ in the presence of a magnetic field, provided that the gas is highly supersonic. If the core is embedded in a cloud with a similar value of $\mathcal{M}_{\mathrm{A}}$ and the cloud is bound, then the magnetic field reduces $\alpha_{\text {vir, }}$ o for both the core and the cloud, and the net effect of the field is small. Hopkins (2012) finds that the critical density depends on the ratio of $\left(c_{\mathrm{S}}^{2}+\sigma^{2}+v_{\mathrm{A}}^{2}\right)$ on the cloud and core scales, so the field does not have a significant effect if $\mathcal{M}_{\mathrm{A}}$ is about the same on the two scales. By contrast, Federrath and Klessen (2012) include magnetic fields in the KM derivation of $\rho_{\text {crit }}$ by generalizing the comparison of Jeans length and sonic length to the comparison of the magnetic Jeans length with the magneto-sonic length. This reduces the $\mathrm{KM}$ value by a factor $\left(1+\beta^{-1}\right)$, where $\beta$ is the ratio of thermal to magnetic pressure, in agreement with the PN model (see Table 1). For the HC model, instead, Federrath and Klessen (2012) find that the critical density increases with increasing magnetic field strength, because it depends inversely on $\mathcal{M}_{\mathrm{s}, \mathrm{cl}}$ (see eq. 12) rather than directly, as in the KM and PN models. These contradictory 
results on the effect of the magnetic field are discussed in more detail in McKee et al. (2014, in preparation).

\subsection{The Star Formation Rate}

The dimensionless star formation rate per free-fall time, $\mathrm{SFR}_{\mathrm{ff}}$, is defined as the fraction of cloud (or clump) mass converted into stars per cloud mean free-fall time, $t_{\mathrm{ff}, 0}=$ $\sqrt{3 \pi / 32 G \rho_{0}}$, where $\rho_{0}$ is the mean density of the cloud:

$$
\operatorname{SFR}_{\mathrm{ff}}(t)=\frac{\dot{M}_{*}(t)}{M_{\mathrm{cl}}(t)} t_{\mathrm{ff}, 0},
$$

where all quantities are in principle time-dependent.

Detailed comparisons between the KM, PN and $\mathrm{HC}$ models have been presented in Hennebelle and Chabrier (2011) and in Federrath and Klessen (2012) and will only be summarized here. A more general formalism to derive the SFR has been developed by Hopkins (2013), but as this author does not provide explicit formulae for the SFR, it is difficult to include here the results of his model.

The common point between these models is that they all rely on the so-called turbulent fragmentation scenario for star formation, with star formation resulting from a field of initial density fluctuations imprinted by supersonic turbulence. The models assume that gravitationally unstable density fluctuations are created by the supersonic turbulence and thus follow the turbulence density PDF (eq. (4)). The SFR is then derived as the integral of the density PDF above a critical density (the HC model is actually derived from the integral of the mass spectrum of gravitationally unstable density fluctuations, but, after some algebra and simplifying assumptions, it is reduced to an integral of the density PDF above a critical density). The differences between the SFR models result from the choice of the critical density (see Section 3.2 and Table 1), at least when the same general formulation for the SFR is used for all models, as first proposed by Hennebelle and Chabrier (2011) and further explored by Federrath and Klessen (2012):

$$
\operatorname{SFR}_{\mathrm{ff}}=\frac{\epsilon}{\phi_{t}} \int_{\widetilde{\rho}_{\text {crit }}}^{\infty} \frac{t_{\mathrm{ff}, 0}}{t_{\mathrm{ff}}(\widetilde{\rho})} p(\widetilde{\rho}) \mathrm{d} \widetilde{\rho},
$$

where $p(\widetilde{\rho}) \mathrm{d} \widetilde{\rho}=\widetilde{\rho} p(s) d s$, and $p(s) d s$ is given by eq. (4), with the variance following the relations (5) or (6). Notice that, in the HC model, the relations (5) or (6) include a scale dependence reflecting the property of the turbulence velocity power spectrum, $\sigma_{s}(R)=\sigma_{s, 0} f(R)$ (e.g. eqn. (5) in Hennebelle and Chabrier (2008)). As mentioned below, this point is of importance when calculating the SFR.

In (14), the PDF is divided by the free-fall time of each density, because unstable fluctuations are assumed to turn into stars in a free-fall time. In steady state, the shape of the density PDF should be constant with time and thus the turbulent flow must replenish density fluctuations of any amplitude. This may require a time longer that the free-fall time at a given density, hence the replenishment factor, $\phi_{t}$, is introduced. It may in general be a function of $\widetilde{\rho}$, but given our poor understanding of the replenishment process, it is simply assumed to be constant. Hennebelle and Chabrier (2011) suggested to use the turbulence turnover time as an estimate of the replenishment time. They showed (in their Appendix) that this choice yields $\phi_{t} \approx 3 . \mathrm{KM}$ assumed that the replenishment time was of order the global free-fall time, not the local one, and their factor $\phi_{t}$ was defined accordingly. PN assumed that the replenishment time was of order the free-fall time of the critical density, and did not introduce a parameter $\phi_{t}$ to parameterize the uncertainty of this choice. Fitting each theory to numerical simulations, Federrath and Klessen (2012) find that $\phi_{t} \approx 2-5$ in the best-fit models (see Figure 3 below).

Note that the factor $t_{\mathrm{ff}, 0} / t_{\mathrm{ff}}(\rho)$ appears inside the integral (14) because gas with different densities has different freefall times, which must be taken into account in the most general case (see Hennebelle and Chabrier, 2011). Previous estimates for $\mathrm{SFR}_{\mathrm{ff}}$ either used a factor $t_{\mathrm{ff}, 0} / t_{\mathrm{ff}, 0}=1$ (Krumholz and McKee, 2005), or a factor $t_{\mathrm{ff}, 0} / t_{\mathrm{ff}}\left(\rho_{\text {crit }}\right)$ (Padoan and Nordlund, 2011), both of which are independent of density and were thus pulled out of the general integral, Equation (14). We refer to the latter models as 'single freefall' models, while Equation (14) is a 'multi-freefall' model, but we can still distinguish three different density thresholds $s_{\text {crit }}=\ln \left(\widetilde{\rho}_{\text {crit }}\right)$ based on the different assumptions in the $\mathrm{KM}, \mathrm{PN}$, and $\mathrm{HC}$ theories. The general solution of Equation (14) is

$$
\mathrm{SFR}_{\mathrm{ff}}=\frac{\epsilon}{2 \phi_{t}} \exp \left(\frac{3}{8} \sigma_{s}^{2}\right)\left[1+\operatorname{erf}\left(\frac{\sigma_{s}^{2}-s_{\text {crit }}}{\sqrt{2 \sigma_{s}^{2}}}\right)\right],
$$

and depends only on $s_{\text {crit }}$ and $\sigma_{s}$, which in turn depend on the four basic, dimensionless parameters $\alpha_{\text {vir }}, \mathcal{M}_{\mathrm{s}}, b$, and $\beta$, as shown and derived for all models in Federrath and Klessen (2012) (see Table 1 for a summary of the differences between each theoretical model for the SFR). It must be stressed that integrating analytically eq. (14) to yield eq. (15) is possible only if the variance of the PDF does not entail a scale dependence. In the HC formalism, this corresponds to what these authors refer to as their "simplified" multi-freefall theory (section 2.4 of Hennebelle and Chabrier (2011)), while their complete theory properly accounts for the scale dependence.

The coefficient $\epsilon$ accounts for two efficiency factors: the fraction of the mass with density larger than $\rho_{\text {crit }}$ that can actually collapse (a piece of very dense gas may be too small or too turbulent to collapse) and the fraction that ends up into actual stars (the so called core-to-star efficiency). The first factor should already be accounted for in the HC derivation of eq. (14), and therefore should not be incorporated into $\epsilon$. However, given the simplifications in the derivation, and the uncertainty in choosing the extreme of integration of the mass spectrum mentioned above $(\S 3.2)$, it may be necessary to allow for a fraction of this efficiency factor to be included in $\epsilon$. The second factor (the coreto-star efficiency) is partly constrained by observations as 
Table 1: Six Analytical Models for the Star Formation Rate per Freefall Time.

\begin{tabular}{llll}
\hline \hline Analytic Model & Freefall-time Factor & Critical Density $\rho_{\text {crit }} / \rho_{0}=\exp \left(s_{\text {crit }}\right)$ & SFR \\
\hline KM & 1 & $\left(\pi^{2} / 45\right) \phi_{x}^{2} \times \alpha_{\mathrm{cl}} \mathcal{M}_{\mathrm{s}}^{2}\left(1+\beta^{-1}\right)^{-1}$ & $\epsilon /\left(2 \phi_{t}\right)\left\{1+\operatorname{erf}\left[\left(\sigma_{s}^{2}-2 s_{\text {crit }}\right) /\left(8 \sigma_{s}^{2}\right)^{1 / 2}\right]\right\}$ \\
PN & $t_{\mathrm{ff}}\left(\rho_{0}\right) / t_{\mathrm{ff}}\left(\rho_{\mathrm{crit}}\right)$ & $(0.067) \theta^{-2} \times \alpha_{\mathrm{cl}} \mathcal{M}_{\mathrm{s}}^{2} f(\beta)$ & $\epsilon /\left(2 \phi_{t}\right)\left\{1+\operatorname{erf}\left[\left(\sigma_{s}^{2}-2 s_{\text {crit }}\right) /\left(8 \sigma_{s}^{2}\right)^{1 / 2}\right]\right\} \exp \left[(1 / 2) s_{\mathrm{crit}}\right]$ \\
$\mathrm{HC}$ & $t_{\mathrm{ff}}\left(\rho_{0}\right) / t_{\mathrm{ff}}(\rho)$ & $\left(\pi^{2} / 5\right) y_{\mathrm{cut}}^{-2} \times \alpha_{\mathrm{cl}} \mathcal{M}_{\mathrm{s}}^{-2}\left(1+\beta^{-1}\right)+\tilde{\rho}_{\text {crit }, \text { turb }}$ & $\epsilon /\left(2 \phi_{t}\right)\left\{1+\operatorname{erf}\left[\left(\sigma_{s}^{2}-s_{\text {crit }}\right) /\left(2 \sigma_{s}^{2}\right)^{1 / 2}\right]\right\} \exp \left[(3 / 8) \sigma_{s}^{2}\right]$ \\
multi-ff KM & $t_{\mathrm{ff}}\left(\rho_{0}\right) / t_{\mathrm{ff}}(\rho)$ & $\left(\pi^{2} / 5\right) \phi_{x}^{2} \times \alpha_{\mathrm{cl}} \mathcal{M}_{\mathrm{s}}^{2}\left(1+\beta^{-1}\right)^{-1}$ & $\epsilon /\left(2 \phi_{t}\right)\left\{1+\operatorname{erf}\left[\left(\sigma_{s}^{2}-s_{\text {crit }}\right) /\left(2 \sigma_{s}^{2}\right)^{1 / 2}\right]\right\} \exp \left[(3 / 8) \sigma_{s}^{2}\right]$ \\
multi-ff PN & $t_{\mathrm{ff}}\left(\rho_{0}\right) / t_{\mathrm{ff}}(\rho)$ & $(0.067) \theta^{-2} \times \alpha_{\mathrm{cl}} \mathcal{M}_{\mathrm{s}}^{2} f(\beta)$ & $\epsilon /\left(2 \phi_{t}\right)\left\{1+\operatorname{erf}\left[\left(\sigma_{s}^{2}-s_{\text {crit }}\right) /\left(2 \sigma_{s}^{2}\right)^{1 / 2}\right]\right\} \exp \left[(3 / 8) \sigma_{s}^{2}\right]$ \\
multi-ff HC & $t_{\mathrm{ff}}\left(\rho_{0}\right) / t_{\mathrm{ff}}(\rho)$ & $\left(\pi^{2} / 5\right) y_{\text {cut }}^{-2} \times \alpha_{\mathrm{cl}} \mathcal{M}_{\mathrm{s}}^{-2}\left(1+\beta^{-1}\right)$ & $\epsilon /\left(2 \phi_{t}\right)\left\{1+\operatorname{erf}\left[\left(\sigma_{s}^{2}-s_{\text {crit }}\right) /\left(2 \sigma_{s}^{2}\right)^{1 / 2}\right]\right\} \exp \left[(3 / 8) \sigma_{s}^{2}\right]$ \\
\hline
\end{tabular}

Notes. Note that the critical density for the KM and multi-ff KM models were derived in Krumholz and McKee (2005) based on the 1D velocity dispersion, while Federrath and Klessen (2012) used the 3D velocity dispersion, which changes the coefficient from $\left(\pi^{2} / 45\right) \phi_{x}^{2}$ to $\left(\pi^{2} / 5\right) \phi_{x}^{2}$. The function $f(\beta)$, entering the critical density in the PN and multi-ff PN models is given in Padoan and Nordlund (2011). The added turbulent contribution $\tilde{\rho}_{\text {crit,turb }}$ in the critical density of the HC model is given in Hennebelle and Chabrier (2011), Federrath and Klessen (2012), and Eq. (12). For further details, see Federrath and Klessen (2012), from which this table was taken.

well as analytical calculations and simulations, suggesting a value $\epsilon \simeq 0.3-0.7$ (e.g. Matzner and McKee, 2000; Federrath and Klessen, 2012, 2013). As in the case of $\phi_{t}, \epsilon$ is assumed to be a constant, even if, in principle, it may have a density dependence. To be consistent with their physical meaning, the values of these two parameters should satisfy the conditions $\phi_{t} \geq 1$ and $\epsilon \leq 1$.

As first proposed by Hennebelle and Chabrier (2011), the KM and PN models should be extended based on eq. (14), which is easily done by inserting the KM and PN expressions for $\rho_{\text {crit }}$ in that equation. The original KM and PN models were equivalent to a simplified version of eq. (14), where one substitutes $t_{\mathrm{ff}}(\widetilde{\rho})$ with $t_{\mathrm{ff}, 0}(\mathrm{KM})$ or $t_{\mathrm{ff}}\left(\widetilde{\rho}_{\text {crit }}\right)(\mathrm{PN})$. The effect of this extension is significant in the case of the KM model, because it introduces a dependence of $\mathrm{SFR}_{\mathrm{ff}}$ on $\mathcal{M}_{\mathrm{s}}$ that is otherwise missing, but rather small in the case of the PN model, where that dependence was already present. Federrath and Klessen (2012) showed that this extension from a single-freefall to a multi-freefall theory of the SFR does improve the match of the KM and PN models with their simulations (see $\S 4.4$ ).

\section{SIMULATIONS}

Numerical simulations where physical conditions may be easily varied and controlled, can greatly contribute, both qualitatively and quantitatively, to our understanding of the most important factors influencing the star formation rate. In this Section, we first discuss numerical methods, then present results from large parameter studies.

\subsection{Numerical Methods}

SFRs measured in numerical experiments should be primarily influenced by the fundamental numerical parameters, such as Mach numbers, virial numbers, types of external driving, etc. However, shortcomings in the numerical representation of turbulence, on the one hand, and specific details (or lack thereof) in the recipes used to implement accretion onto 'sink particles' representing the real stars may also influence the results significantly.
Some of the general shortcomings, particularly in relation to the need to reproduce a turbulent cascade covering a wide range of scales, were reviewed in Klein et al. (2007). Since then, comparisons of numerical codes have been presented by Kitsionas et al. (2009) for the case of decaying supersonic HD turbulence, and by Kritsuk et al. (2011a) for the case of decaying supersonic MHD turbulence. Price and Federrath (2010) compared grid and particle methods for the case of driven, supersonic HD turbulence.

From the point of view of the current discussion, the bottom line of these comparisons is that the effective resolution of the various grid-based codes used in the most recent SFR measurements are quite similar, and that a much larger range of resolution results from the use (or not) of AMR than from the use of different codes. On problems where direct comparisons can be made (mainly HD problems) gridand particle-based codes also show similar results. Even though these problems do not have analytical solutions that may be used for verification, the general behavior of both $\mathrm{HD}$ and MHD turbulence is well represented by the codes that have been used to empirically measure the SFR.

An important point to keep in mind, however, is that both the Kitsionas et al. (2009) and Kritsuk et al. (2011a) comparisons, as well as Federrath et al. (2010a) and Federrath et al. (2011a), illustrate that turbulent structures are resolved well only down to a scale of 10-30 grid cells; smaller scales are, to quote Kitsionas et al. (2009), "significantly affected by the specific implementation of the algorithm for solving the equations of hydrodynamics". These effects, as well as the relative similarity with which different codes are affected, are well illustrated by Fig. 3 of Kritsuk et al. (2011a), which shows that a drop in compensated velocity power spectra sets in about one order of magnitude below the Nyquist wave number; i.e., at about 20 cells per wavelength, in essentially all codes. Even the world's highestresolution simulation of supersonic turbulence to date, with $4096^{3}$ grid cells, only yields a rather limited inertial range (Federrath, 2013a). The possible influence of the magnetic Prandtl number, effectively close to unity in numerical experiments, while typically much larger in the ISM, should 
also be kept in mind (Federrath et al., 2011b; Schober et al., 2012; Bovino et al., 2013; Schleicher et al., 2013).

In numerical experiments, the SFR is measured using 'sink particles' that can accumulate gas from their surroundings. The use of sink particles is convenient, because one can directly measure the conversion of gas mass into stellar mass. It is also inevitable, since it would otherwise be very difficult to handle the mass that undergoes gravitational collapse. The sink-particle technique goes back to Boss and Black (1982), who used a single, central 'sink cell' in a grid code. Since then, sink-particle methods have been described and discussed e.g. by Bate et al. (1995), Krumholz et al. (2004), Jappsen et al. (2005), and by Federrath et al. (2010b).

Bate et al. (1995) applied a sink-particle technique to the modeling of accretion in protobinary systems, using an SPH method, with a series of criteria for deciding when to dynamically form new sink particles. The most important criteria are that the density exceeds a certain, fixed threshold, and that the total energy is negative; i.e., the gas is bound. To decide whether to accrete onto existing sink particles they used a fixed accretion radius, within which an SPH particle would be accreted if it was gravitationally bound, with an angular momentum implying a circular orbit less than the accretion radius, and with auxiliary conditions imposed to resolve ambiguous cases.

Krumholz et al. (2004) applied a similar method in the context of an AMR grid code (Orion). To decide when to create new sink particles they used primarily the local Jeans length, which is equivalent to a density threshold criterion. Sink particles were created when a Jeans length was resolved with less than four grid cells, thus combining the sink-particle creation criterion with the Truelove criterion (Truelove et al., 1997), which prevents artificial gravitational fragmentation. To handle the creation of very close sink particles they merged all sink particles with distances below a given accretion radius. To decide how rapidly to accrete onto existing sink particles they used a method based primarily on the Bondi-Hoyle accretion formula, complemented by taking the effects of residual angular momentum into account.

Federrath et al. (2010b) implemented sink particles in a version of the Flash code (Fryxell et al., 2000; Dubey et al., 2008), using a similar series of criteria as in Bate et al. (1995) to guard against for example spurious creation in shocks. They checked the resulting behavior in the AMR case against the behavior in the original SPH case. They demonstrated that omitting some of the sink-creation criteria may result in significantly overestimating the number of stars created, as well as overestimating the SFR.

Krumholz et al. (2004), Federrath et al. (2010b), and Haugbolle et al. (2013, in preparation) seem to agree on the fact that the global SFR in simulations is not as sensitive to details of the sink-particle recipes as the number and mass distribution of sink particles. It appears that the rate of accretion is determined at some relatively moderate density level, well below typical values adopted as thresholds for sink-particle creation, and that various auxiliary criteria can result in the formation of a larger or smaller number of sinks, without affecting the global SFR very much.

An important class of effects that are largely missing from current simulations aimed at measuring the SFR consists of (direct and indirect) effects of stellar feedback. Bipolar outflows, for example, both reduce the final mass of the stars (a 'direct' effect), and feed kinetic energy back into the ISM, thus increasing the virial number and reducing the SFR (an 'indirect' effect).

As discussed elsewhere in this Chapter, both comparisons of the core mass function with the stellar initial mass function and comparisons of numerical simulations with observations indicate that of the order of half of the mass is lost in bipolar outflows, i.e., leading to a core-to-star efficiency $\epsilon \approx 0.3-0.7$ (Matzner and McKee, 2000; Federrath and Klessen, 2012). In the near future, it may become possible to model the outflow in sufficient detail to measure the extent to which accretion is diverted into outflows, using local zoom-in with very deep AMR simulations, which are able to resolve the launching of outflows in the immediate neighborhood of sink particles (Seifried et al., 2012; Price et al., 2012; Nordlund et al., 2013).

The indirect effect of outflow feedback has been modelled by adopting assumptions about the mass and momentum loading of bipolar outflows (e.g. Nakamura and Li, 2008; Wang et al., 2010; Cunningham et al., 2011). When attempting to quantify the importance of the feedback, it is important to adopt realistic values of the virial number. If the virial number of the initial setup is small, the relative importance of the feedback from outflows is increased. A number of studies of molecular clouds have found very extended inertial ranges (e.g., Ossenkopf and Mac Low, 2002; Heyer and Brunt, 2004; Padoan et al., 2006; Roman-Duval et al., 2011), and relatively smooth velocity power spectra without bumps or features at high wavenumbers (e.g. Padoan et al., 2009; Brunt et al., 2009), suggesting that local feedbacks are not a dominant energy source, compared to the inertial energy cascade from very large scales.

Finally, a few comments on the importance of feedback from stellar radiation (see the Chapters by Krumholz et al. and Offner et al. in this book). As obvious from a number of well known Hubble images, and also from simple backof-the-envelope estimates, the UV radiation from new-born massive stars can have dramatic effects on the surrounding ISM, ionizing large ISM bubbles and cooking away the outer layers of cold, dusty molecular clouds. Such effects have recently started to become incorporated in models of star formation (e.g. Peters et al., 2010; Commerçon et al., 2011; Kuiper et al., 2011; Krumholz et al., 2012b; Bate, 2012). In a series of papers, Dale et al. (2012b,a, 2013b,a) have explored the effect of HII regions on the SFR. They find that the ionization feedback reduces the SFR, by an amount that depends on the virial parameter and total mass of the cloud. To derive a realistic measure of the effect of feedbacks, one needs to make sure that other factors that affect the SFR have realistic levels as well. For example, 
most numerical studies of the effect of massive star feedback have neglected magnetic fields, which are known to reduce the SFR by a factor of two or three (Padoan and Nordlund, 2011; Federrath and Klessen, 2012, and $\S 4.3$ ) even without any stellar feedback. Furthermore, as real clouds are generally not isolated, inertial driving from larger scales (for example gas accretion onto a cloud, see e.g., Klessen and Hennebelle, 2010; Schmidt et al., 2013) may also reduce the SFR.

\subsection{Setups of Numerical Experiments}

Besides the differences in numerical methods discussed in the previous section, numerical studies of star formation may also differ significantly with respect to their boundary and initial conditions. Ideally, one would like to model the whole process starting from cloud formation in galaxy simulations (e.g., Tasker and Bryan, 2006; Dobbs et al., 2006, see also the chapter by Dobbs et al. in this book) and large-scale colliding flows (Heitsch et al., 2005; VázquezSemadeni et al., 2006; Hennebelle et al., 2008; Banerjee et al., 2009) down to the formation of individual stars. However, this is beyond the current capabilities of supercomputers. Due to their local nature (typically $\sim 1-10 \mathrm{pc}$ ), star formation simulations do not couple the formation of prestellar cores and their collapse with the large-scale processes driving the ISM turbulence, such as the evolution of supernova ( $\mathrm{SN}$ ) remnants, and the formation and evolution of giant molecular clouds (GMCs). In setting up such simulations, one can choose either to completely ignore the effect of the larger scales (e.g. Clark et al., 2005; Bate, 2009; Bonnell et al., 2011; Girichidis et al., 2011), or to model it with external forces (e.g. Klessen et al., 2000; Heitsch et al., 2001; Padoan and Nordlund, 2011; Padoan et al., 2012; Federrath and Klessen, 2012).

When driving forces are included, it is usually done with periodic boundary conditions and with a random, largescale force generated in Fourier space. This artificial random force is supposed to mimic a turbulent cascade of energy starting at a much larger scale, but its effect may be different from that of the true driving forces of the ISM, such as SNs, spiral arm compression, expanding radiation fronts, winds, jets, outflows, and other mechanisms. The SFR in the simulations depends on whether a driving force is included or not (e.g. Klessen et al., 2000; Vázquez-Semadeni et al., 2003). Without an external force, the turbulence decays rapidly (Padoan and Nordlund, 1997; Stone et al., 1998; Mac Low et al., 1998; Padoan and Nordlund, 1999; Mac Low, 1999) and star formation proceeds at a very high rate, while, if the turbulence is continuously driven, a lower SFR can be maintained for an extended time. Both types of numerical setups have drawbacks. If external forces are neglected and star formation proceeds very rapidly, unphysical initial conditions may strongly affect the results; if the turbulence is driven, initial conditions are forgotten over time, but the results may depend on the artificial force.

Large differences between simulations are also found in their initial conditions. These can be taken to be rather artificial, for example with stochastic velocity fields inconsistent with supersonic turbulence apart from their power spectrum (e.g. Clark et al., 2008; Bate, 2009), or selfconsistently developed by driving the turbulence over many dynamical times prior to the inclusion of self-gravity (e.g. Offner et al., 2009; Padoan and Nordlund, 2011; Padoan et al., 2012; Federrath and Klessen, 2012). In some studies, initial conditions are taken to follow ad hoc density profiles or velocity power spectra with the purpose of studying the effect of those choices (e.g. Bate and Bonnell, 2005; Girichidis et al., 2011).

\subsection{Results from Large Parameter Studies}

Some of the first numerical studies testing the effects of the turbulence, its driving scale and the magnetic field strength on the SFR were carried out by Klessen et al. (2000), Heitsch et al. (2001), and Vázquez-Semadeni et al. (2003). Recent high-resolution simulations by Padoan and Nordlund (2011), Padoan et al. (2012) and Federrath and Klessen (2012), covering the largest range of molecular cloud parameters available to date, demonstrate that the SFR is primarily determined by four dimensionless parameters:

1. the virial parameter, $\alpha_{\mathrm{vir}}=2 E_{\mathrm{kin}} / E_{\mathrm{grav}}$,

2. the sonic Mach number, $\mathcal{M}_{\mathrm{s}}=\sigma_{v} / c_{\mathrm{s}}$,

3. the turbulence driving parameter, $b$ in Equations (5) and (6), and

4. the plasma $\beta=\left\langle P_{\text {gas }}\right\rangle /\left\langle P_{\operatorname{mag}}\right\rangle$, or the Alfvénic Mach number $\mathcal{M}_{\mathrm{A}}$, defined as $\mathcal{M}_{\mathrm{A}}=\sigma_{v} / v_{\mathrm{A}}=$ $\sigma_{v} /\left(\left\langle B^{2}\right\rangle /\langle 4 \pi \rho\rangle\right)^{1 / 2}$.

These parameter studies adopted periodic boundary conditions, an isothermal equation of state, and large-scale random driving. The turbulence was driven without selfgravity for several dynamical times, to reach a statistical steady state. Self-gravity was then included, and collapsing regions above a density threshold, $\rho_{\max }$, and satisfying other conditions (see Section 4.1), were captured by sink particles.

Padoan and Nordlund (2011) carried out the first large parameter study of the SFR using the Stagger-code to run simulations on uniform numerical grids of $500^{3}$ and $1000^{3}$ computational points. They created the sink particles only based on a density threshold, $\rho_{\max }=8000\langle\rho\rangle$, large enough to avoid non-collapsing density fluctuations created by shocks in the turbulent flow. They explored two different values of $\mathcal{M}_{\mathrm{s}}$, two different values of $\beta$, and seven different values of $\alpha_{\text {vir }}$. Their simulations showed that $\mathrm{SFR}_{\mathrm{ff}}$ can be reduced by the magnetic field by approximately a factor of three, that in the non-magnetized case $\mathrm{SFR}_{\mathrm{ff}}$ increases with increasing values of $\mathcal{M}_{\mathrm{s}}$, and that, both with and without magnetic fields, $\mathrm{SFR}_{\mathrm{ff}}$ decreases with increasing values of $\alpha_{\text {vir }}$, confirming the predictions of their model. 


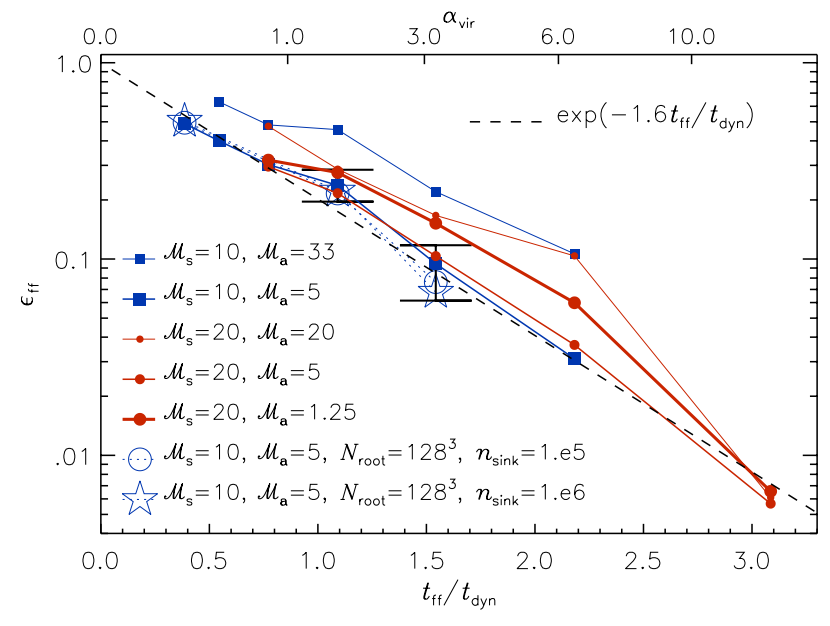

Fig. 2.- SFR per free-fall time, $\mathrm{SFR}_{\mathrm{ff}}$, versus $t_{\mathrm{ff}} / t_{\mathrm{dyn}}$ (bottom abscissa) and $\alpha_{\text {vir }}$ (top abscissa). The symbols for each series of runs, where only the strength of gravity is changed and $\mathcal{M}_{\mathrm{s}}$ and $\mathcal{M}_{\mathrm{A}}$ are kept constant, are connected by a line, to better distinguish each series. The two error bars give the mean of $\mathrm{SFR}_{\mathrm{ff}}$, plus and minus the rms values, for each group of five $32^{3}$-root-grid runs with identical parameters $\left(\mathcal{M}_{\mathrm{s}} \approx 10\right.$ and $\left.\mathcal{M}_{\mathrm{A}} \approx 5\right)$, but different initial conditions. The dashed line is an approximate exponential fit to the minimum value of $S F R_{\mathrm{ff}}$ versus $t_{\mathrm{ff}} / t_{\mathrm{dyn}}$. From Padoan et al. (2012), reproduced by permission of the AAS.

In their following work, Padoan et al. (2012) analyzed an even larger parameter study, based on 45 AMR simulations with the Ramses code, with a maximum resolution equivalent to $32,768^{3}$ computational points. Thanks to the very large dynamic range, they could adopt a threshold density for the creation of sink particles of $\rho_{\max }=10^{5}\langle\rho\rangle$, much larger than in the uniform-grid simulations. The creation of a sink particle also required that the cell was at a minimum of the gravitational potential, and that the velocity divergence was negative. They explored two values of sonic Mach number, $\mathcal{M}_{\mathrm{s}}=10$ and 20, four values of initial Alfvénic Mach number, $\mathcal{M}_{\mathrm{A}}=1.25,5,20$, 33 , and seven values of the virial parameter, in the approximate range $0.2<\alpha_{\mathrm{vir}}<20$. Their results are presented in Figure 2, and can be summarized in three points: i) $\mathrm{SFR}_{\mathrm{ff}}$ decreases exponentially with increasing $t_{\mathrm{ff}} / t_{\mathrm{dyn}}$ $\left(\propto \alpha_{\text {vir }}^{1 / 2}\right.$ ), but is insensitive to changes in $\mathcal{M}_{\mathrm{s}}$ (in the range $10 \leq \mathcal{M}_{\mathrm{s}} \leq 20$ ), for constant values of $t_{\mathrm{ff}} / t_{\mathrm{dyn}}$ and $\mathcal{M}_{\mathrm{A}}$. ii) Decreasing values of $\mathcal{M}_{\mathrm{A}}$ (increasing magnetic field strength) reduce $\mathrm{SFR}_{\mathrm{ff}}$, but only to a point, beyond which $\mathrm{SFR}_{\mathrm{ff}}$ increases with a further decrease of $\mathcal{M}_{\mathrm{A}}$. iii) For values of $\mathcal{M}_{\mathrm{A}}$ characteristic of star-forming regions, $\mathrm{SFR}_{\mathrm{ff}}$ varies with $\mathcal{M}_{\mathrm{A}}$ by less than a factor of two. Therefore, Padoan et al. (2012) proposed a simple law for the SFR depending only on $t_{\mathrm{ff}} / t_{\mathrm{dyn}}$, based on the empirical fit to the minimum SFR ${ }_{\mathrm{ff}}: \mathrm{SFR}_{\mathrm{ff}} \approx \epsilon \exp \left(-1.6 t_{\mathrm{ff}} / t_{\mathrm{dyn}}\right)$ (dashed line in Figure 2), where $\epsilon$ is the core-to-star formation efficiency.
These results were confirmed and extended in a third parameter study by Federrath and Klessen (2012), based on 34 uniform grid simulations with the Flash code, using a resolution of up to $512^{3}$ computational points (plus one AMR run with maximum resolution equivalent to $1,024^{3}$ computational points). Six of their runs include a magnetic field, covering the range $1.3 \leq \mathcal{M}_{\mathrm{A}} \leq 13$, but all with approximately the same sonic Mach number, $\mathcal{M}_{\mathrm{s}} \approx 10$, so the lack of dependence of $\mathrm{SFR}_{\mathrm{ff}}$ on $\mathcal{M}_{\mathrm{s}}$ found by Padoan et al. (2012) could not be verified. On the other hand, the 28 runs without magnetic fields span a wide range of values of sonic Mach number, $2.9 \leq \mathcal{M}_{\mathrm{s}} \leq 52$, which allowed Federrath and Klessen (2012) to confirm the analytical and numerical result of Padoan and Nordlund (2011), that in the non-magnetized case $\mathrm{SFR}_{\mathrm{ff}}$ increases with increasing $\mathcal{M}_{\mathrm{s}}$. Federrath and Klessen (2012) also studied the effect of varying $b$ (the ratio of compressible to total energy in the turbulence driving), and carried out a systematic comparison of their simulations with the predictions of SFR models and observations (see Sections 4.4 and 5.1, respectively).

Federrath and Klessen (2012) found that both $\mathcal{M}_{\mathrm{s}}$ and $b$ can introduce order-of-magnitude variations in $\mathrm{SFR}_{\mathrm{ff}}$, in the absence of magnetic fields. Increasing $b$ and $\mathcal{M}_{\mathrm{s}}$ produces a wider density PDF (see eqs. 5 and 6) and thus pushes a larger fraction of gas above the critical density for star formation, increasing $\mathrm{SFR}_{\mathrm{ff}}$ (a larger $\mathcal{M}_{\mathrm{s}}$ results in a larger critical density, but also in a shorter free-fall time at such density). For purely compressive driving, Federrath and Klessen (2012) found a $4 \times$ higher $\mathrm{SFR}_{\mathrm{ff}}$, when increasing $\mathcal{M}_{\mathrm{s}}$ from 5 to 50 . For fixed $\mathcal{M}_{\mathrm{s}}=10$, which is a reasonable average Mach number for Milky Way clouds, they found that purely compressive (curl-free, $b=1$ ) driving yields about $10 \times$ higher $\mathrm{SFR}_{\mathrm{ff}}$ compared to purely solenoidal (divergence-free, $b=1 / 3$ ) driving. The increase of $\mathrm{SFR}_{\mathrm{ff}}$ for compressive driving is caused by the denser structures (filaments and cores) produced with such driving, which are more gravitationally bound than the structures produced with purely solenoidal driving.

Increasing the magnetic field strength and thus decreasing $\beta$, reduces $\mathrm{SFR}_{\mathrm{ff}}$. Numerical simulations by Padoan and Nordlund (2011), Padoan et al. (2012) and by Federrath and Klessen (2012) quantify the effect of the magnetic field and find a maximum reduction of the SFR by a factor of 2-3 in strongly magnetized, trans- to sub-Alfvénic turbulence compared to purely hydrodynamic turbulence. This is a relatively small change in $\mathrm{SFR}_{\mathrm{ff}}$ compared to changes induced by $\alpha_{\mathrm{vir}}, \mathcal{M}_{\mathrm{s}}$, and $b$, in the absence of magnetic fields.

\subsection{Comparison with Theoretical Models}

To compare them with numerical results, we separate the theories of the SFR into six cases (see Section 3.3), named 'KM', 'PN', 'HC', and 'multi-ff KM', 'multi-ff PN', 'multiff HC', following the notation in Hennebelle and Chabrier (2011) and Federrath and Klessen (2012). The first three represent the original analytical derivations by Krumholz and McKee (2005), Padoan and Nordlund (2011), and Hen- 

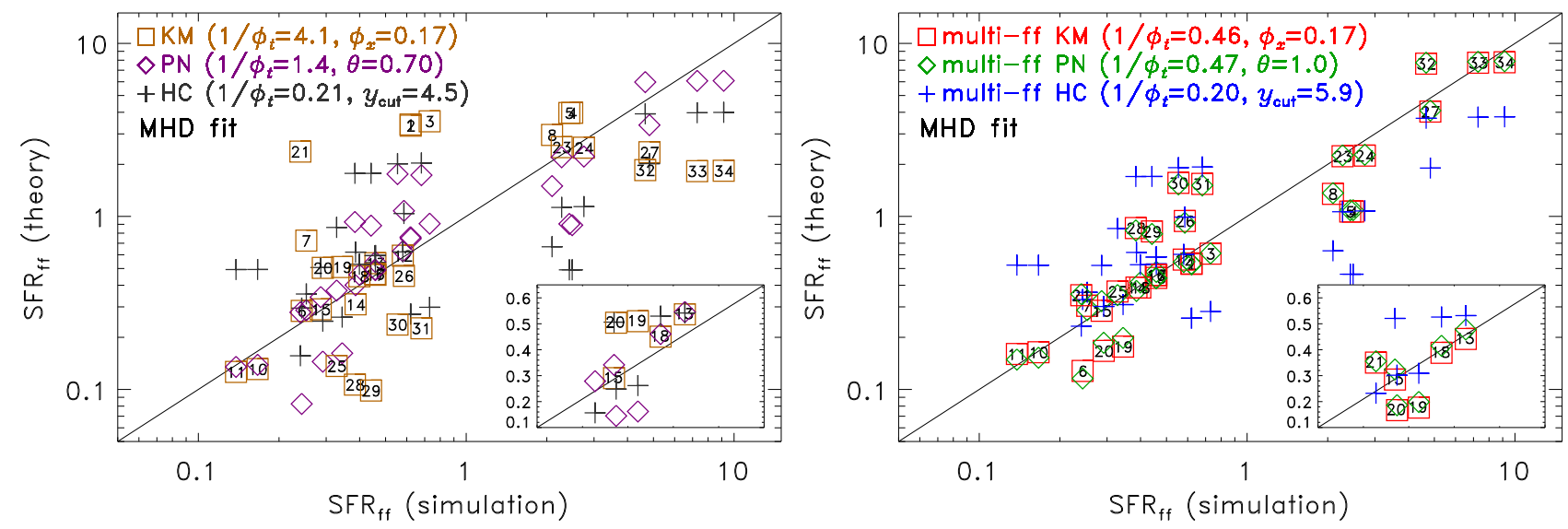

Fig. 3.- Comparison of $\mathrm{SFR}_{\mathrm{ff}}$ (theory) with $\mathrm{SFR}_{\mathrm{ff}}$ (simulation). The left panel shows the original KM (boxes), PN (diamonds), and $\mathrm{HC}$ (crosses) theories, while the right panel shows the multi-freefall version of each theory defined in Hennebelle and Chabrier (2011). The multi-freefall prescription is superior to all single-freefall models and provides good fits to the numerical simulations (the insets show blow-ups of the MHD simulations; the x-range of the insets is identical to the y-range). The multi-ff KM and multi-ff PN models agree to within a factor of three with any of the numerical simulations over the two orders of magnitude in SFRs tested. The simulation number in Table 2 in Federrath and Klessen (2012) is given in the boxes for each $\mathrm{SFR}_{\mathrm{ff}}$ (simulation). From Federrath and Klessen (2012), reproduced by permission of the AAS.

nebelle and Chabrier (2011), while the last set of three are all based on the multi-freefall expression of eq. (14). Notice that the HC models, here and in Federrath and Klessen (2012), refer to the aforementioned simplified version of the $\mathrm{HC}$ model without a scale dependence in the density variance, which yields eq. (15). In case of the complete theory, the integral of eq. (14) can no longer be performed analytically. Besides this important point, the difference among the multi-freefall models is the expression for the critical density (the lower limit of the integral in eq. 14). Table 1 summarizes the differences among all six theories following Federrath and Klessen (2012), who also extended all theoretical models to include the effects of magnetic pressure (except PN, where this dependence was already included).

The comparison between the theoretical and the numerical $\mathrm{SFR}_{\mathrm{ff}}$ is shown in Figure 3 (left panels: KM, PN, $\mathrm{HC}$; right panels: multi-freefall KM, PN, HC). Any of the multi-freefall theories provides significantly better fits to the numerical simulations than the single-freefall models $\left(\chi_{\text {red }}^{2}=1.3\right.$ and 1.2 for multi-ff KM and PN; $\chi_{\text {red }}^{2}=5.7$ and 1.8 for $\mathrm{KM}$ and $\mathrm{PN}$ ). Note that $\mathrm{HC}$ and multi-ff $\mathrm{HC}$ are both multi-ff models, but Hennebelle and Chabrier (2011) defined them this way to distinguish their model where the critical density includes both thermal and turbulent support (called HC) from the one where only thermal support is included (called multi-ff HC) (see Table 1 and $\S 3.2$ ).

The simplified HC model, although originally designed as a multi-freefall model, does not fit the simulations as well as the multi-ff KM and multi-ff PN models. This is likely related to the definition of the critical density in $\mathrm{HC}$, where it scales with $\mathcal{M}_{\mathrm{s}}^{-2}$, while in $\mathrm{KM}$ and PN it scales with $\mathcal{M}_{\mathrm{s}}^{2}$ (see Section 3.2, Table 1, and the derivation in Federrath and Klessen (2012)). A caveat of the present comparison is that the density PDF in the models is assumed to be perfectly log-normal. Accounting for deviations of the PDF in future semi-analytical models may further improve the agreement with the simulations.

Figure 3 shows that the multi-freefall KM and PN theories provide good fits to the numerical simulations. However, some data points in Figure 3 deviate from the theoretical predictions by a factor of $2-3$. The simulations that deviate the most have relatively low numerical resolution. A careful resolution study of these models shows that they converge to the diagonal line in Figure 3. Notably, simulation models 32,33 , and 34 with numerical resolutions of $256^{3}, 512^{3}$, and $1024^{3}$ grid cells (in the upper right corner of Figure 3) clearly converge to the diagonal line with increasing resolution. The same is true for the MHD models 19 and 20 with $256^{3}$ and $512^{3}$ resolution in the inset of Figure 3. This convergence with increasing resolution supports the conclusion that the multi-freefall theories for the SFR provide a good match to numerical simulations where the interplay of self-gravity and supersonic turbulence is the primary process controlling the SFR.

\section{THEORY AND SIMULATIONS VERSUS OBSER- VATIONS}

\subsection{Model Predictions versus Observed SFR}

A comparison of simulations from Federrath and Klessen (2012) with observations of $\Sigma_{\mathrm{SFR}}$ in Milky Way clouds by Heiderman et al. (2010) is shown in Figure 4. The observational data are from Galactic observations of clouds and young stellar objects (YSOs) identified in the Spitzer Cores-to-Disks (c2d) and Gould's-Belt (GB) surveys (Evans et al., 2009), of massive dense clumps (Gao 


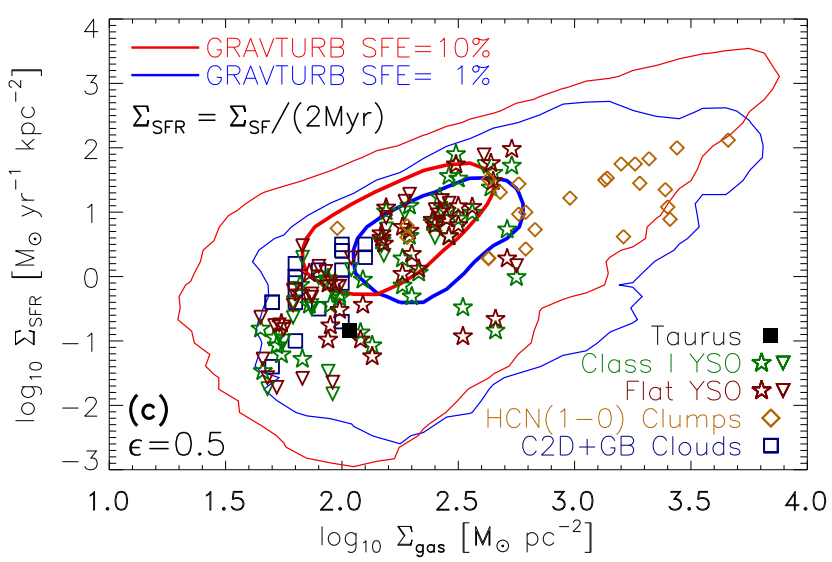

Fig. 4.- Star formation rate column density $\Sigma_{\mathrm{SFR}}$ versus gas column density $\Sigma_{\text {gas }}$ for Milky Way clouds compiled in Heiderman et al. (2010) (symbols) and in the GRAVTURB simulations by Federrath and Klessen (2012) (contours). Individual data points are Taurus: filled black box (data from Goldsmith et al., 2008; Pineda et al., 2010; Rebull et al., 2010), Class I YSOs and Flat YSOs: green and red stars and upper-limits shown as downward-pointing triangles, $\mathrm{HCN}(1-0)$ Clumps: golden diamonds (data from Gao and Solomon, 2004; Wu et al., 2005, 2010), and C2D+GB Clouds: dark blue boxes (data from Evans et al., 2009). Blue and red contours show data from numerical simulations by Federrath and Klessen (2012) for a star formation efficiency $\mathrm{SFE}=1 \%$ (blue) and SFE $=10 \%$ (red). The thick contours enclose $50 \%$ of all $\left(\Sigma_{\text {gas }}, \Sigma_{\mathrm{SFR}}\right)$ simulation pairs, while the thin contours enclose $99 \%$. All simulation data were scaled to a local core-to-star efficiency of $\epsilon=0.5$ (Matzner and McKee, 2000), providing the best fit to the observational data. From Federrath and Klessen (2012), reproduced by permission of the AAS.

and Solomon, 2004; Wu et al., 2005, 2010), and of the Taurus molecular cloud (Goldsmith et al., 2008; Pineda et al., 2010; Rebull et al., 2010). Simulation data are shown as contours for SFE $=1 \%$ (blue) and $\mathrm{SFE}=10 \%$ (red).

The simulation data from Federrath and Klessen (2012) shown as contours in Figure 4 were transformed into the observational space by measuring $\Sigma_{\text {gas }}$ and $\Sigma_{\mathrm{SFR}}$ with a method as close as possible to what observers do to infer $\Sigma_{\mathrm{SFR}}$-to- $\Sigma_{\text {gas }}$ relations (see, e.g., Bigiel et al., 2008; Heiderman et al., 2010), including the effects of telescope beam smoothing. This was done by computing twodimensional projections of the gas column density, $\Sigma_{\text {gas }}$, and the sink particle column density, $\Sigma_{\mathrm{SF}}$, along each coordinate axis: $x, y, z$. Each of these 2D maps was smoothed to a resolution $\left(N_{\text {res }} / 8\right)^{2}$ for a given 3D numerical resolution $N_{\text {res }}^{3}=128^{3}-1024^{3}$ grid cells (for a complete list of simulations and their parameters, see Table 2 in Federrath and Klessen, 2012), such that the size of each pixel in the smoothed maps slightly exceeds the sink particle diameter (which is 5 grid cells). We then search for pixels with a sink particle column density greater than zero, $\Sigma_{\mathrm{SF}}>0$, and extract the corresponding pixel in the gas column density map, which gives $\Sigma_{\text {gas }}$ in units of $M_{\odot} \mathrm{pc}^{-2}$ for that pixel. The SFR column density is computed by taking the sink particle column density $\Sigma_{\mathrm{SF}}$ of the same pixel and dividing it by a characteristic timescale for star formation, $t_{\mathrm{SF}}$, which yields $\Sigma_{\mathrm{SFR}}=\Sigma_{\mathrm{SF}} / t_{\mathrm{SF}}$ in units of $M_{\odot} \mathrm{yr}^{-1} \mathrm{kpc}^{-2}$. The simplest choice for $t_{\mathrm{SF}}$ is a fixed star formation time, $t_{\mathrm{SF}}=2 \mathrm{Myr}$, based on an estimate of the elapsed time between star formation and the end of the Class II phase (e.g., Evans et al., 2009; Covey et al., 2010). This is also the $t_{\mathrm{SF}}$ adopted by Lada et al. (2010) and Heiderman et al. (2010) to convert young stellar object (YSO) counts into an SFR column density (see Section 2.4), so we used it here as the standard approach. However, Federrath and Klessen (2012) also experimented with two other choices for $t_{\mathrm{SF}}$, which gave similar results, considering the large dispersion of the observational data.

The Heiderman et al. (2010) sample of SFR column densities for Galactic clouds in Figure 4 covers different evolutionary stages of the clouds, such that a single SFE for the whole sample is unlikely. However, we can reasonably assume SFEs in the range 1\% to $10 \%$ (Evans et al., 2009; Federrath and Klessen, 2013). For a typical SFE $=3 \%$, Federrath and Klessen (2012) find a best-fit match of their simulations to the Heiderman et al. (2010) sample by scaling the simulations to a core-to-star efficiency $\epsilon=0.5$, in agreement with theoretical results (Matzner and McKee, 2000), with observations of jets and outflows (Beuther et al., 2002), and with independent numerical simulations that concentrate on the collapse of individual cloud cores (e.g., Wang et al., 2010; Seifried et al., 2012).

The simulation data in Figure 4 are also consistent with the Milky Way cloud samples in Lada et al. (2010) and Gutermuth et al. (2011), showing that $\Sigma_{\mathrm{SFR}}$ can vary by more than an order of magnitude for any given $\Sigma_{\text {gas }}$. These variations can be explained by variations in $\alpha_{\text {vir }}$ (Hennebelle and Chabrier, 2011; Padoan et al., 2012), $\mathcal{M}_{\mathrm{s}}$ (Renaud et al., 2012; Federrath, 2013b), b (Federrath and Klessen, 2012), and $\beta$ (Padoan and Nordlund, 2011; Federrath and Klessen, 2012) from cloud to cloud. Besides these physical effects primarily related to the statistics of self-gravitating turbulence, the $\Sigma_{\mathrm{SFR}}-\Sigma_{\text {gas }}$ relation is also somewhat affected by observational issues (e.g., telescope resolution; see Heiderman et al., 2010; Federrath and Klessen, 2012) and by variations in the metallicity (Krumholz et al., 2012a). The latter effect introduces uncertainties by a factor of about two (Glover and Clark, 2012).

Figure 5 compares the SFR obtained with the complete $\mathrm{HC}$ formalism for four different cloud sizes $R_{c}=0.5,2$, 5 and $20 \mathrm{pc}$, with the data from Heiderman et al. (2010) and Gutermuth et al. (2011), for the case of both isothermal and non-isothermal gas, for $y_{c u t}=0.25$ and $b=0.5$ (approximate equipartition between solenoidal and compressive modes of turbulence). For such typical Milky Way molecular cloud conditions, the agreement is fairly reasonable. The observed scatter, with no one-to-one correspondence between the SFR and the surface density, is well 

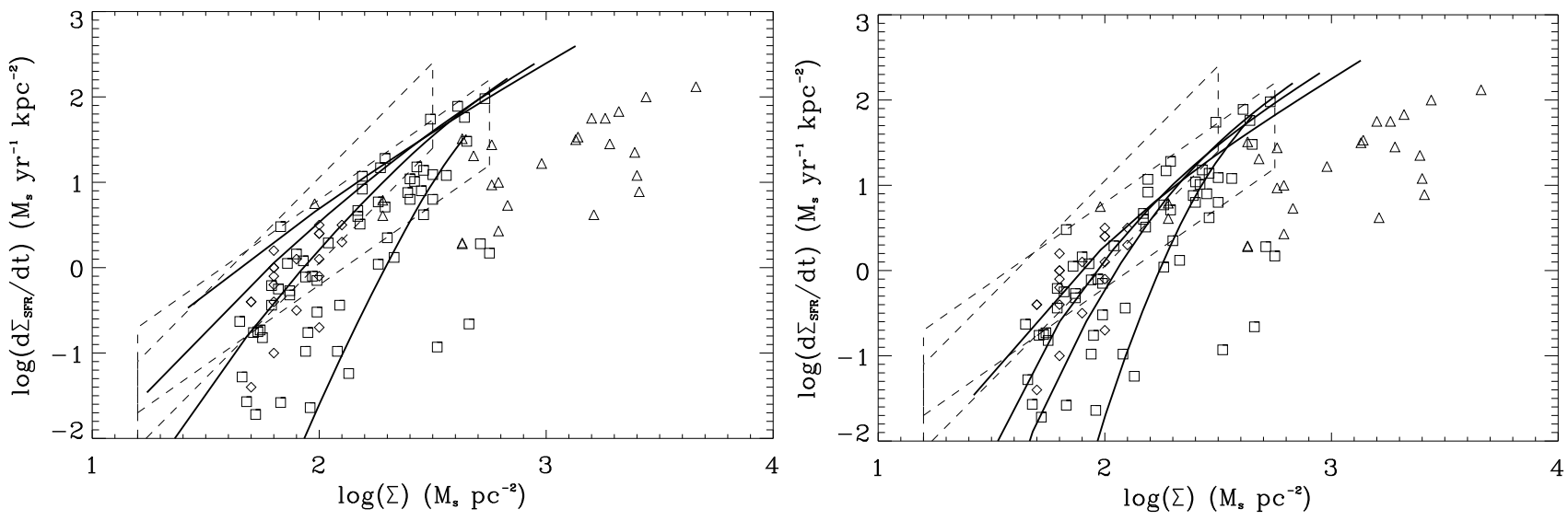

Fig. 5.- Star formation rate per unit area, $\Sigma_{\mathrm{SFR}}$, as a function of gas surface density, $\Sigma_{\text {gas }}$. Solid lines: results obtained with the complete HC SFR theory (Hennebelle and Chabrier, 2013) for cloud sizes $R_{c}=20,5,2$ and $0.5 \mathrm{pc}$, from left to right, in the case of isothermal (left panel) and non-isothermal (right panel) gas, for $y_{c u t}=0.25$ and $b=0.5$, and assuming a density-size relation, $\rho \propto R_{c}^{-0.7}$. Each curve is obtained by varying the normalization of the density-size relation (see Hennebelle and Chabrier (2013) for details). The data correspond to observational determinations by Heiderman et al. (2010) for massive clumps (triangles) and molecular cloud YSOs (diamonds+squares), and by Gutermuth et al. (2011) (bracketed areas). From Hennebelle and Chabrier (2013), reproduced by permission of the AAS.

explained by the strong dependence of the SFR upon the cloud's size/mass, besides the effect of the turbulence parameters. The theory also adequately reproduces the observational SFR values of Gutermuth et al. (2011) (bracketed areas) for the adequate (large) cloud sizes, down to the very low density regime, $\Sigma \sim 30 \mathrm{M}_{\odot} \mathrm{pc}^{-2}$. This supports the suggestion that there is no real step-function threshold condition for star formation and that the latter can occur even in rather low-density regions, although at a much lower rate.

In Figure 5, the $\mathrm{HC}$ model is applied assuming a densitysize relation, $\rho \propto R_{c}^{-0.7}$, which determines the dependence on $\Sigma_{\text {gas }}$ seen in the theoretical curves. This density-size relation is confirmed by observations where clouds are defined by constant ${ }^{13} \mathrm{CO}$ brightness contours (Roman-Duval et al., 2010). However, when clouds are defined by constant surface density contours, one gets a different densitysize relation, $\rho \propto R_{c}^{-1.0}$ (Lombardi et al., 2010), which changes the position of the theoretical curves of Figure 5 slightly (the curves would be a bit more vertical and closer to one another). This highlights the importance of adopting unambiguous definitions of MCs in both theoretical models and observational works, or rather of deriving theoretical models that do not depend on MC definitions.

\subsection{Density PDF in MCs}

Several studies have tried to compare the PDF predicted by supersonic turbulence with measurements in MCs. The pdf of a MC was first shown to be consistent with a lognormal function by Padoan et al. (1997a). They used the extinction measurements of IC 5146 by Lada et al. (1994), specifically their $\sigma_{A_{\mathrm{V}}}-A_{\mathrm{V}}$ relation, to constrain the shape and standard deviation of the three-dimensional density PDF, and the density power spectrum. They concluded that the density PDF was consistent with a log-normal, that the standard deviation was $\sigma_{\widetilde{\rho}}=5.0 \pm 0.5$, that the slope of the density power spectrum was $-2.6 \pm 0.5$, and that density fluctuations had to reach at least a scale of $0.03 \mathrm{pc}$. Given the Mach number derived for IC 5146, $\mathcal{M}_{\mathrm{s}} \approx 10$, these results were all in good agreement with their numerical simulations of super-Alfvénic turbulence, showing that $\sigma_{\widetilde{\rho}}=b \mathcal{M}_{\mathrm{s}}$, with $b \approx 0.5$.

Brunt et al. (2010b) derived analytically the relation between the variance of the three-dimensional density field and the observed variance and power spectrum of the projected density. Applied to the Taurus MC, this method gave a density variance yielding $b=0.48_{-0.11}^{+0.15}$, similar to the case of IC 5146, and consistent with that of turbulence simulations driven with a mixture of solenoidal and compressional modes (Brunt, 2010; Price et al., 2011).

The relation between the Mach numbers and the variance of the projected density can be derived directly from simulations. Burkhart and Lazarian (2012) found that the variance, $\sigma_{S}$, of the logarithm of the column density, $S=\ln \left(\Sigma / \Sigma_{0}\right)$ (where $\Sigma_{0}$ is the mean column density), depends on the sonic Mach number, and follows the relation $\sigma_{S}^{2}=A \ln \left[1+b^{2} \mathcal{M}_{\mathrm{s}}^{2}\right]$, where $A \approx 0.11$, for a range of values of Mach numbers, $0.4 \leq \mathcal{M}_{s} \leq 8.8$ and $0.3 \leq \mathcal{M}_{\mathrm{A}} \leq 3.2$. They also used that relation to map the Mach number in the Small Magellanic Cloud (Burkhart et al., 2010). By comparing with eq. (5), one gets that the ratio of density and column density variances is independent of the Mach number, $\sigma_{S}^{2} / \sigma_{s}^{2}=A$. In a subsequent paper, Burkhart et al. (2013) studied the role of radiative transfer effects on the derivation of the column density variance using molecular emission lines. They showed that the parameter $A$ is quite sensitive to the optical depth of the observed line, so extinction maps, rather than molecular emis- 
sion lines, are the method of choice to directly constrain the density PDF, unless opacities and molecular abundances are known. Similar conclusions about the uncertainties in deriving column density PDFs in MCs with $\mathrm{CO}$ emission lines were also reached by Goodman et al. (2009), through a comparison of column density measured with dust emission and extinction. They also found that the relation between the mean and the variance of the projected density were consistent with a log-normal distribution.

Besides deriving the density variance, a more ambitious goal is to derive the whole PDF using MC observations. This was first done by Padoan et al. (1999), who compared a simulation of supersonic and super-Alfvénic turbulence with a ${ }^{13} \mathrm{CO}$ survey of the Perseus region. Radiative transfer effects were properly accounted for, because the comparison was done using synthetic observations of the simulation, generated with a three-dimensional non-LTE radiative transfer code (Padoan et al., 1998). However, besides radiative transfer, the interpretation of molecular emission lines would also require a careful study of molecular abundances, in order to probe a more extended density range. The interpretation of extinction maps is more straightforward, at least for MCs that are far enough from the galactic plane to avoid confusion, and close enough to guarantee a large number of background stars. Moreover, Brunt et al. (2010a) developed a statistical method to reconstruct the volumetric (3D) density PDF from observations of the column (2D) density PDF and power spectrum.

Extinction maps were used by Kainulainen et al. (2009) to derive column density PDFs of many nearby clouds. They found that the PDFs are consistent with a log-normal function at all densities in non-star-forming clouds. In regions of active star formation, the PDF is log-normal at intermediate and low densities, and a power law at high densities, consistent with the results of supersonic turbulence with self-gravity. Other studies based on extinction maps used the cumulative PDF of column density, and found again deviations from the log-normal at high extinctions in regions of active star formation (Froebrich and Rowles, 2010; Kainulainen et al., 2013a). The relation between extinction-based cumulative PDF of column density and the SFR was derived even more explicitly by Lada et al. (2010), who found a linear correlation between the SFR and the mass of gas with extinction $A_{K}>0.8 \mathrm{mag}$.

Recently, FIR dust emission maps from Herschel have also been used to derive the PDF of column density in MCs. For example, Schneider et al. $(2012,2013)$ have shown that PDFs from different regions of the Rosette MC deviate from the log-normal, although the PDF tails are not well described by single power laws. Cumulative column density PDFs from Herschel maps are found to agree with cumulative PDFs from near- and mid-infrared extinction maps, and are therefore a powerful probe of the structure of infrared dark clouds (Kainulainen et al., 2013b).

\subsection{Star Formation Thresholds in MCs}

As already mentioned in Section 2.5, studies of nearby clouds, based on a variety of methods (near-IR dust extinction, sub-mm dust emission, or molecular emission lines) have demonstrated the existence of a column density threshold for star formation of approximately $120 M_{\odot} \mathrm{pc}^{-2}$, below which star formation appears to be rare. This threshold should not be interpreted as a strict step-function condition for star formation (presumably, the SFE continues to increase at larger $\Sigma_{\text {gas }}$, up to a maximum equal to $\epsilon$, the core-to-star efficiency, when the characteristic $\Sigma_{\text {gas }}$ of cores is reached-see Section 2.5). The idea of the threshold is to exclude the large mass of the cloud that is currently not forming stars. Understanding how to stop this mass from eventually forming stars lies at the heart of the SFE problem for clouds and galaxies. The correlation between the mass fraction of gas with surface density above the threshold and the SFR/area found by Heiderman et al. (2010) and Lada et al. (2010) may become even stronger at even larger column densities (Gutermuth et al., 2011). This is suggested for example by the increase in the SFE toward smaller (denser) scales (Federrath and Klessen, 2013), with the mass in dense cores being comparable to the mass in YSOs (Enoch et al., 2008; Jorgensen et al., 2008).

It is nevertheless interesting to contrast the observed threshold with the critical volume density of the SFR models, $\rho_{\text {crit }}$. Indeed, Lada et al. (2010) have argued that the column density threshold they found could also be interpreted as a volume density threshold of approximately $10^{4} \mathrm{~cm}^{-3}$, and the equivalent mean volume density within the column density threshold for the $\mathrm{c} 2 \mathrm{~d}+\mathrm{GB}$ clouds is about $6000 \mathrm{~cm}^{-3}$ (Evans et al., in preparation). The mass per unit length threshold for star formation found by André et al. (2010) in MC filaments probed by Herschel can also be viewed both as a column or volume density threshold, because the filaments they select have a well defined characteristic thickness, independent of their surface density ( $\mathrm{Ar}$ zoumanian et al., 2011, 2013).

We have demonstrated in Section 3.2 that the general expression for the theoretical critical density, eq. (11), as well as the equivalent expressions from the KM model, eq. (8) and the PN model, eq. (9), yield a constant value, $n_{\mathrm{H} \text {, crit }}=3.0 \times 10^{4} \mathrm{~cm}^{-3}$ (see eq. 10). This is not true for the $\mathrm{HC}$ model, where the critical density, given by eq. (12), depends on the cloud size (the larger the cloud the smaller the critical density), even after assuming a standard linewidth-size relation. We conclude that, in the case of the KM and PN models, one would expect observational signatures of a star formation threshold approximately independent of cloud properties, at least within a MC sample following approximately the same velocity-size relation. Based on the $\mathrm{HC}$ model, instead, one would expect the threshold to be approximately the same only for MCs of the same size, and to increase toward smaller cloud sizes.

The observed threshold of Lada et al. (2010), $10^{4} \mathrm{~cm}^{-3}$, is a few times smaller than the above value of $n_{\mathrm{H} \text {, crit }}$. However, as mentioned above, it is not a strict step-function condition for star formation, and it is to be expected that 
the correlation between the SFR and the gas mass fraction above the threshold would become increasingly stronger as the observed threshold is increased toward the theoretical value. If the theoretical value were reached, then the SFR per unit mass should be approximately the mass fraction above $n_{\mathrm{H}}$, crit divided by the free-fall time of $n_{\mathrm{H}}$, crit , multiplied by the local core-to-star efficiency. In other words, most of the gas denser than $n_{\mathrm{H} \text {, crit }}$ should be collapsing or about to start to do so.

The prediction for high-mass star-forming cores would probably be different than for local MCs. The cores in the samples of Plume et al. (1997) and Shirley et al. (2003), for example, have rms velocities well in excess of the Larson relations for nearby clouds. Assuming that their virial parameter is not very different from that of nearby clouds, their larger rms Mach number at a given size (their warmer temperature probably does not compensate the increased linewidth) would imply a larger value of $n_{\mathrm{H}}$, crit than in nearby clouds. On the other hand, if the HC model were correct, the threshold density in these regions should not increase with the Mach number.

\section{CONCLUSIONS AND FUTURE DIRECTIONS}

\subsection{Summary and Conclusions}

Progress on theory, simulations, and observations of star formation has been substantial since Protostars \& Planets V. We have summarized this progress, focusing on the observational and theoretical estimates of the SFR in individual clouds, with some reference to larger-scale star formation on galaxy scales.

Observationally (Section 2) we now have extinction maps of nearby clouds, defining cloud structures, surface densities, and masses, with far less uncertainty than was inherent in molecular line maps. Most of the cloud mass lies at low surface densities, well below $100 \mathrm{M}_{\odot} \mathrm{pc}^{-2}$. The surface densities follow a log-normal PDF at low extinctions ( $A_{V}<$ few mag), but clouds with active star formation show power law tails. Maps of dust continuum emission show that the denser parts of clouds are highly filamentary, with dense cores lying along filaments containing only a very small fraction of the cloud mass. We also have quite complete censuses of YSOs in all the larger nearby $(d<500 \mathrm{pc})$ molecular clouds, allowing robust estimates of SFRs averaged over the half-life of infrared excesses ( $\sim 2 \mathrm{Myr}$ ). These are quite low compared to what would be expected if clouds were collapsing at the free-fall rate, calculated from the mean cloud density, and making stars with unity efficiency; $\mathrm{SFR}_{\mathrm{ff}} \sim 0.01$ for the clouds as a whole, but the scatter is large. If attention is focused on regions above a surface density "threshold" of about $120 \mathrm{M}_{\odot} \mathrm{pc}^{-2}$, the dispersion in SFR per mass of this "dense" gas is much smaller, essentially consistent with observational uncertainties, and the mean $\mathrm{SFR}_{\mathrm{ff}}$ doubles to $\sim 0.02$. Thus, most of the mass of nearby clouds is quite inactive in star formation. The mean depletion time of the nearby clouds is about 140
Myr; if only $10 \%$ of the mass forms clouds that form stars before dissipating, the galaxy scale depletion time of about 1-2 Gyr can result from these characteristic values of local efficiency: star formation may thus be seen as the ultimate outcome of an 'inefficient hierarchy'.

The fact that star formation is slow compared to freefall times, and ultimately inefficient (mass depletion times are much greater than structure lifetimes) may be understood from current theories (Section 3) as essentially a consequence of an excess of the turbulent kinetic plus magnetic energies over the gravitational energy of a cloud. This understanding has come about as a consequence of numerical efforts that resulted in large parameter studies based on many simulations (Section 4). A number of formalisms addressing the problem of estimating the SFR in clouds with magnetized turbulence have been developed. Their predictions have been checked against both idealized simulations (Section 4) of such clouds, and against observations (Section 5). While formulations differ in detail, the differences have been narrowed down and clarified in recent papers, as discussed in this review.

As indicated by the theoretical models (Section 3), and illustrated in practice by numerical simulations (Section 4), in addition to the virial ratio there are a number of other factors that also influence the SFR; among them the sonic and Alfvénic Mach numbers, and the relative importance of compressive and incompressive components of the random forces driving the turbulence. These parameters are all included in the current theoretical models for the SFR, and the best theories can successfully predict the SFR within a factor of 2-3 (Figure 3), over at least two orders of magnitude in SFR and for a wide range of parameter space, which is very encouraging, given the simplified assumptions in the analytical theories (most importantly, the current assumption of a purely log-normal PDF together with a volumedensity threshold for star formation). The importance of radiative feedback from early type stars is also broadly appreciated and accepted, as is the possible importance of kinetic feedback from low-mass stars (see the Chapter by Krumholz et al. in this book). These latter aspects are, however, very difficult to incorporate into the analytical theories, and are also non-trivial and computationally costly to include in numerical simulations. We thus expect further developments of both analytical and numerical models addressing these issues in the near future

\subsection{Future Directions}

An important future step will be to apply what we have learned here about the SFR in the Milky Way to distant galaxies at low and high redshift, including starburst galaxies. Extragalactic studies focus primarily on KennicuttSchmidt-type relations, i.e., measuring the SFR column density, $\Sigma_{\mathrm{SFR}}$, as a function of gas column density, $\Sigma_{\text {gas }}$ (e.g., Kennicutt, 1998b; Bigiel et al., 2008; Kennicutt and Evans, 2012), like in Figure 4.

In a recent theoretical effort with comparison to observa- 
tions, Krumholz et al. (2012a) suggested a unification of star formation in the Milky Way and in distant galaxies at low and high redshift into a universal law where $\Sigma_{\mathrm{SFR}}$ is about $1 \%$ of the gas collapse rate, $\Sigma_{\text {gas }} / t_{\mathrm{ff}}$. This model is purely empirical, but fits observations with $\Sigma_{\mathrm{SFR}}$ varying over five orders of magnitude quite well. Yet, a physical foundation of this empirical model is still missing; it must of course ultimately be a result of the physical laws governing star formation that we have discussed in this review, with the apparent uniformity a result of a corresponding uniformity of the particular combination of physical properties that determine the SFR. The remaining scatter in $\Sigma_{\mathrm{SFR}}$ around the proposed universal law is likely to reflect, in addition to an unavoidable observational scatter, local statistical fluctuations in physical properties.

Adding more observational data, including all local cloud and YSO measurements from Heiderman et al. (2010), Gutermuth et al. (2011), the Central Molecular Zone (Yusef-Zadeh et al., 2009), and the Small Magellanic Cloud (Bolatto et al., 2011) in addition to the lowand high-redshift disk and starburst galaxies, Federrath (2013b) confirms the relatively tight correlation of $\Sigma_{\text {SFR }}$ with $\Sigma_{\text {gas }} / t_{\text {ff }}$ (compared to $\Sigma_{\text {gas }}$ only) and also suggests a likely contribution to the scatter in this correlation. Federrath (2013b) included simulation data with different forcing of the turbulence, sonic Mach numbers ranging from 5-50, and varying magnetic field strength in the Krumholz et al. (2012a) framework, showing that a significant fraction of the scatter might be explained by variations in the properties of the turbulence, in particular by variations in the sonic Mach number of the star-forming clouds. Applying the theoretical models for the local SFR derived and discussed in this chapter, Federrath (2013b) shows that they are consistent with the simulation data and with the notion that the scatter seen in the observations can be explained by variations in the parameters of the turbulence. To test this suggestion, it will be necessary to measure the parameters of the turbulence, in particular the virial parameter, the sonic and Alfvén Mach numbers, the driving parameter $b$, and the star formation efficiency of the star-forming portions of all the objects contributing to the $\Sigma_{\mathrm{SFR}}-\Sigma_{\text {gas }} / t_{\mathrm{ff}}$ relation.

Specific tasks for future studies include:

1) Clarify definitions of MCs, both from the point of view of observations alone, and, more importantly, for establishing a better basis for comparing simulations with observations. This will become easier when using larger-scale simulations, where clouds can be selected using essentially the same methods as in observations.

2) Investigate the dense-gas mass fraction of starforming clouds as a direct test of theoretical predictions (irrespective of a determination of the SFR). This could include a detailed numerical and observational study of the relation between the critical density of SFR theories, and the density where the PDF departs from the log-normal.

3) Compare numerical simulations with observations avoiding the (necessarily very uncertain) 'inversion' of ob- servations into fundamental parameters, and focusing instead on the use of statistical 'fingerprints' that can be readily constructed from observations and simulations alike. These may include spatial correlation functions of cores or YSOs, as well as column density PDFs.

4) Determine how to relate the SFR from counting YSOs in local clouds to indirect methods for more distant clouds and to global averages, using extragalactic methods. In such an effort, which aims at 'calibrating' the extragalactic methods, the use of 'forward analysis' based on numerical simulations (constructing synthetic observations from simulations) may constitute a useful tool.

5) Extend studies of the SFR versus gas properties to regions forming massive stars and clusters. These typically are denser and more turbulent and may reveal the importance of parameters like Mach number and turbulent forcing. Because they are more distant, different techniques are needed to study both the cloud structure and the star formation properties. From the modeling perspective they also pose challenges, because chemistry and radiative energy transfer is needed for a quantitative comparison with observations.

6) Explore even larger scales, for greater variety of SF conditions, to avoid modeling star-forming clouds as isolated systems with artificial boundary and initial conditions, and to study the role of large-scale feedbacks and driving. This will be achieved with higher-resolution extragalactic observations adequately interpreted (Kruijssen and Longmore, 2013), and with multi-scale star-formation simulations (whole galaxies or galactic fountains) that account for realistic driving forces such as galactic dynamics, SN explosions, stellar radiation and stellar outflows (Mac Low and Klessen, 2004).

Ultimately, the point of view taken in most of this review (as well as in the research papers reviewed), namely to explore and understand how the SFR depends on fundamental physical properties of the ISM, needs to be combined with the complementary view: asking how these fundamental properties of the ISM are, in turn, affected by star formation, and to what extent they are also modulated and affected by other external factors, such as galactic dynamics and density waves.

Given that we already know, from numerical simulations and theoretical modeling consistent with the simulations, that the SFR is a very steep function of the virial parameter, in particular at SFR values as low as those that are observed, one conclusion is practically unavoidable: there must be a strong feedback from the average SFR level back to the physical properties that, in turn, determine the SFR. If one assumed, hypothetically, that the SFR was entirely controlled by external factors, with for example the normalization of the turbulent cascade (the constant factor in Larson's velocity relation) being entirely determined by galactic dynamics, with no influence of the feedback from star formation, that would be tantamount to claiming that the rather universal observed $\mathrm{SFR}_{\mathrm{ff}}$ of $\sim 1 \%$ was a coincidental outcome of these external agents, maintaining a suffi- 
ciently uniform virial parameter for the SFR to come out right (or else manipulating also other variables to serendipitously compensate for virial parameter variations). This appears much less likely than to assume that the feedback from stars closes a 'servo loop', which keeps the SFR at a value that corresponds to consistency between the integrated feedback (SN explosions, outflows, and radiation) generated by star formation, and the distribution of physical parameters (particularly the virial parameter) that these feedbacks can sustain.

External factors such as galactic dynamics are surely present, but star formation must play an important role for this servo loop to be effective. A possible problem with this idea is that the feedback is much stronger and different in regions forming massive stars, yet the star formation rate per free-fall time is similar. However, the existence of a pervasive inertial range in velocity dispersion, covering a large range of scales, shows that, whatever the ultimate source of the driving, the mechanical energy is distributed rather efficiently in space, being thus able to power also low mass star formation. The relative uniformity of the SFR may be just another consequence of this. Determining the ratio of contributions from stellar feedback relative to the total driving, and the resulting quasi-stable state of the feedback loop, via a combination of numerical and theoretical modeling, with the eventual success gauged by the consistency with observations, must indeed be considered as the ultimate goal of research into star formation and the SFR. When the fundamental properties of the ISM, such as the (distribution of) virial parameters, Mach numbers, and driving modes come out self-consistently from such modeling, with essentially no free parameters, then that goal has been reached.

Acknowledgments. We thank Ralf Klessen, the anonymous referee, Patrick Hennebelle, Alexei Kritsuk, and Mark Krumholz for reading the manuscript and providing useful comments. PP is supported by the FP7-PEOPLE2010-RG grant PIRG07-GA-2010- 261359. Simulations by PP were carried out on the NASA/Ames Pleiades supercomputer, and under the PRACE project pra50751 running on SuperMUC at the LRZ (project ID pr86li). CF thanks for support from the Australian Research Council for a Discovery Projects Fellowship (Grant DP110102191). NJE was supported by NSF Grant AST-1109116 to the University of Texas at Austin. The research of CFM is supported in part by NSF grant AST-1211729 and NASA grant NNX13AB84G. DJ is supported by the National Research Council of Canada and by a Natural Sciences and Engineering Research Council of Canada (NSERC) Discovery Grant. JKJ is supported by a Lundbeck Foundation Junior Group Leader Fellowship. Research at Centre for Star and Planet Formation was funded by the Danish National Research Foundation and the University of Copenhagens Programme of Excellence. Supercomputing time at Leibniz Rechenzentrum (PRACE projects pr86li, pr89mu, and project pr32lo), at Forschungszentrum Jülich (project hhd20), and at DeIC/KU in Copenhagen are gratefully acknowledged.

\section{REFERENCES}

Agertz O. et al. (2013) Astrophys. J., 770, 25.

Aguirre J. E. et al. (2011) Astrophys. J. Suppl., 192, 4.

Aluie H. (2011) Physical Review Letters, 106, 17, 174502.

Aluie H. (2013) Physica D Nonlinear Phenomena, 247, 54.

Alves J. et al. (2007) Astron. Astrophys., 462, L17.

André P. et al. (2010) Astron. Astrophys., 518, L102.

Arzoumanian D. et al. (2011) Astron. Astrophys., 529, L6.

Arzoumanian D. et al. (2013) Astron. Astrophys., 553, A119.

Ascenso J. et al. (2012) Astron. Astrophys., 540, A139.

Ascenso J. et al. (2013) Astron. Astrophys., 549, A135.

Ballesteros-Paredes J. et al. (2007) Protostars and Planets V, pp. 63-80.

Banerjee R. et al. (2009) Mon. Not. R. Astron. Soc., 398, 1082.

Bate M. R. (2009) Mon. Not. R. Astron. Soc., 392, 590.

Bate M. R. (2012) Mon. Not. R. Astron. Soc., 419, 3115.

Bate M. R. and Bonnell I. A. (2005) Mon. Not. R. Astron. Soc., 356, 1201.

Bate M. R. et al. (1995) Mon. Not. R. Astron. Soc., 277, 362.

Bertoldi F. and McKee C. F. (1992) Astrophys. J., 395, 140.

Bertram E. et al. (2012) Mon. Not. R. Astron. Soc., 420, 3163.

Beuther H. et al. (2002) Astron. Astrophys., 383, 892.

Bigiel F. et al. (2008) Astron. J., 136, 2846.

Bigiel F. et al. (2010) Astron. J., 140, 1194.

Blaisdell G. A. et al. (1993) Journal of Fluid Mechanics, 256, 443.

Bolatto A. D. et al. (2011) Astrophys. J., 741, 12.

Bonnell I. A. et al. (2011) Mon. Not. R. Astron. Soc., 410, 2339.

Boss A. P. and Black D. C. (1982) Astrophys. J., 258, 270.

Bovino S. et al. (2013) New Journal of Physics, 15, 1, 013055.

Brunt C. M. (2010) Astron. Astrophys., 513, A67.

Brunt C. M. et al. (2009) Astron. Astrophys., 504, 883.

Brunt C. M. et al. (2010a) Mon. Not. R. Astron. Soc., 405, L56.

Brunt C. M. et al. (2010b) Mon. Not. R. Astron. Soc., 403, 1507.

Burkhart B. and Lazarian A. (2012) Astrophys. J. Lett., 755, L19.

Burkhart B. et al. (2010) Astrophys. J., 708, 1204.

Burkhart B. et al. (2013) Astrophys. J., 771, 122.

Cambrésy L. (1999) Astron. Astrophys., 345, 965.

Carpenter J. M. (2000) Astron. J., 120, 3139.

Chabrier G. (2003) PASP, 115, 763.

Chapman N. L. et al. (2009) Astrophys. J., 690, 496.

Cho W. and Kim J. (2011) Mon. Not. R. Astron. Soc., 410, L8.

Chomiuk L. and Povich M. S. (2011) Astron. J., 142, 197.

Clark P. C. et al. (2005) Mon. Not. R. Astron. Soc., 359, 809.

Clark P. C. et al. (2008) Mon. Not. R. Astron. Soc., 386, 3.

Collins D. C. et al. (2011) Astrophys. J., 731, 59.

Collins D. C. et al. (2012) Astrophys. J., 750, 13.

Commerçon B. et al. (2011) Astrophys. J. Lett., 742, L9.

Contreras Y. et al. (2013) Astron. Astrophys., 549, A45.

Covey K. R. et al. (2010) Astrophys. J., 722, 971.

Cunningham A. J. et al. (2011) Astrophys. J., 740, 107.

Daddi E. et al. (2010) Astrophys. J. Lett., 714, L118.

Dale J. E. et al. (2012a) Mon. Not. R. Astron. Soc., 427, 2852.

Dale J. E. et al. (2012b) Mon. Not. R. Astron. Soc., 424, 377.

Dale J. E. et al. (2013a) Mon. Not. R. Astron. Soc., 431, 1062.

Dale J. E. et al. (2013b) Mon. Not. R. Astron. Soc., 430, 234. 
di Francesco J. et al. (2006) in: Revealing the Molecular Universe: One Antenna is Never Enough, vol. 356 of Astronomical Society of the Pacific Conference Series, (edited by D. C. Backer, J. M. Moran, and J. L. Turner), p. 275.

di Francesco J. et al. (2007) Protostars and Planets V, pp. 17-32. Dib S. and Burkert A. (2005) Astrophys. J., 630, 238.

Dobashi K. et al. (2005) PASJ, 57, 1.

Dobbs C. L. et al. (2006) Mon. Not. R. Astron. Soc., 371, 1663.

Draine B. T. (2003) Annu. Rev. Astron. Astrophys., 41, 241.

Dubey A. et al. (2008) in: Numerical Modeling of Space Plasma Flows, vol. 385 of Astronomical Society of the Pacific Conference Series, (edited by N. V. Pogorelov, E. Audit, and G. P. Zank), p. 145.

Dunham M. K. et al. (2011) Astrophys. J., 741, 110.

Dunham M. M. et al. (2013) Astron. J., 145, 94.

Elmegreen B. G. and Scalo J. (2004) Annu. Rev. Astron. Astrophys., 42, 211.

Enoch M. L. et al. (2006) Astrophys. J., 638, 293.

Enoch M. L. et al. (2007) Astrophys. J., 666, 982.

Enoch M. L. et al. (2008) Astrophys. J., 684, 1240.

Evans II N. J. et al. (2009) Astrophys. J. Suppl., 181, 321.

Falkovich G. et al. (2010) Journal of Fluid Mechanics, 644, 465.

Federrath C. (2013a) Mon. Not. R. Astron. Soc., in press (arXiv:1306.3989).

Federrath C. (2013b) Mon. Not. R. Astron. Soc., in press (arXiv:1307.1467).

Federrath C. and Klessen R. S. (2012) Astrophys. J., 761, 156.

Federrath C. and Klessen R. S. (2013) Astrophys. J., 763, 51.

Federrath C. et al. (2008a) Astrophys. J. Lett., 688, L79.

Federrath C. et al. (2008b) Physica Scripta Volume T, 132, 1, 014025.

Federrath C. et al. (2009) Astrophys. J., 692, 364.

Federrath C. et al. (2010a) Astron. Astrophys., 512, A81.

Federrath C. et al. (2010b) Astrophys. J., 713, 269.

Federrath C. et al. (2011a) Astrophys. J., 731, 62.

Federrath C. et al. (2011b) Physical Review Letters, 107, 11, 114504.

Froebrich D. and Rowles J. (2010) Mon. Not. R. Astron. Soc., 406, 1350.

Fryxell B. et al. (2000) Astrophys. J. Suppl., 131, 273.

Galtier S. and Banerjee S. (2011) Physical Review Letters, 107, 13, 134501.

Gao Y. and Solomon P. M. (2004) Astrophys. J., 606, 271.

Genzel R. et al. (2010) Mon. Not. R. Astron. Soc., 407, 2091.

Ginsburg A. et al. (2013) Astrophys. J. Suppl., 208, 14.

Girichidis P. et al. (2011) Mon. Not. R. Astron. Soc., 413, 2741.

Girichidis P. et al. (2013) ArXiv e-prints.

Glover S. C. O. and Clark P. C. (2012) Mon. Not. R. Astron. Soc., 426, 377.

Glover S. C. O. and Mac Low M.-M. (2007) Astrophys. J., 659, 1317.

Glover S. C. O. et al. (2010) Mon. Not. R. Astron. Soc., 404, 2.

Goldsmith P. F. et al. (2008) Astrophys. J., 680, 428.

Goodman A. A. et al. (2009) Astrophys. J., 692, 91.

Gutermuth R. A. et al. (2008) Astrophys. J. Lett., 673, L151.

Gutermuth R. A. et al. (2009) Astrophys. J. Suppl., 184, 18.

Gutermuth R. A. et al. (2011) Astrophys. J., 739, 84.

Harvey P. et al. (2007) Astrophys. J., 663, 1149.

Hatchell J. and Fuller G. A. (2008) Astron. Astrophys., 482, 855.

Heiderman A. et al. (2010) Astrophys. J., 723, 1019.

Heitsch F. et al. (2001) Astrophys. J., 547, 280.

Heitsch F. et al. (2005) Astrophys. J. Lett., 633, L113.
Hennebelle P. and Chabrier G. (2008) Astrophys. J., 684, 395.

Hennebelle P. and Chabrier G. (2011) Astrophys. J. Lett., 743, L29.

Hennebelle P. and Chabrier G. (2013) Astrophys. J., 770, 150.

Hennebelle P. et al. (2008) Astron. Astrophys., 486, L43.

Heyer M. et al. (2009) Astrophys. J., 699, 1092.

Heyer M. H. and Brunt C. M. (2004) Astrophys. J. Lett., 615, L45.

Hopkins P. F. (2012) Mon. Not. R. Astron. Soc., 423, 2016.

Hopkins P. F. (2013) Mon. Not. R. Astron. Soc., 430, 1653.

Hsieh T.-H. and Lai S.-P. (2013) Astrophys. J. Suppl., 205, 5.

Jappsen A.-K. et al. (2005) Astron. Astrophys., 435, 611.

Johnstone D. and Bally J. (1999) Astrophys. J. Lett., 510, L49.

Johnstone D. and Bally J. (2006) Astrophys. J., 653, 383.

Johnstone D. et al. (2004) Astrophys. J. Lett., 611, L45.

Johnstone D. et al. (2005) in: Protostars and Planets V, p. 8485.

Jørgensen J. K. et al. (2008) Astrophys. J., 683, 822.

Kainulainen J. et al. (2009) Astron. Astrophys., 508, L35.

Kainulainen J. et al. (2013a) Astron. Astrophys., 553, L8.

Kainulainen J. et al. (2013b) ArXiv e-prints.

Kennicutt R. C. and Evans N. J. (2012) Annu. Rev. Astron. Astrophys., 50, 531.

Kennicutt Jr. R. C. (1998a) Annu. Rev. Astron. Astrophys., 36, 189.

Kennicutt Jr. R. C. (1998b) Astrophys. J., 498, 541.

Kerton C. R. et al. (2001) Astrophys. J., 552, 601.

Kirk H. et al. (2006) Astrophys. J., 646, 1009.

Kirk J. M. et al. (2013) Mon. Not. R. Astron. Soc., 432, 1424.

Kitsionas S. et al. (2009) Astron. Astrophys., 508, 541.

Klein R. I. et al. (2007) Protostars and Planets V, pp. 99-116.

Kleinmann S. G. (1992) in: Robotic Telescopes in the 1990s, vol. 34 of Astronomical Society of the Pacific Conference Series, (edited by A. V. Filippenko), pp. 203-212.

Klessen R. S. (2000) Astrophys. J., 535, 869.

Klessen R. S. and Hennebelle P. (2010) Astron. Astrophys., 520, A17.

Klessen R. S. et al. (2000) Astrophys. J., 535, 887.

Konstandin L. et al. (2012) Astrophys. J., 761, 149.

Kritsuk A. G. et al. (2007) Astrophys. J., 665, 416.

Kritsuk A. G. et al. (2011a) Astrophys. J., 737, 13.

Kritsuk A. G. et al. (2011b) Astrophys. J. Lett., 727, L20.

Kritsuk A. G. et al. (2013) ArXiv e-prints.

Kruijssen J. M. D. and Longmore S. N. (2013) Mon. Not. R. Astron. Soc..

Krumholz M. R. and McKee C. F. (2005) Astrophys. J., 630, 250.

Krumholz M. R. and Tan J. C. (2007) Astrophys. J., 654, 304.

Krumholz M. R. et al. (2004) Astrophys. J., 611, 399.

Krumholz M. R. et al. (2012a) Astrophys. J., 745, 69.

Krumholz M. R. et al. (2012b) Astrophys. J., 754, 71.

Kryukova E. et al. (2012) Astron. J., 144, 31.

Kuiper R. et al. (2011) Astrophys. J., 732, 20.

Lada C. et al. (2013) ArXiv e-prints.

Lada C. J. and Lada E. A. (2003) Annu. Rev. Astron. Astrophys., $41,57$.

Lada C. J. et al. (1994) Astrophys. J., 429, 694.

Lada C. J. et al. (2010) Astrophys. J., 724, 687.

Lada C. J. et al. (2012) Astrophys. J., 745, 190.

Lemaster M. N. and Stone J. M. (2008) Astrophys. J. Lett., 682, L97.

Lombardi M. and Alves J. (2001) Astron. Astrophys., 377, 1023.

Lombardi M. et al. (2010) Astron. Astrophys., 519, L7.

Lunttila T. et al. (2008) Astrophys. J. Lett., 686, L91.

Lunttila T. et al. (2009) Astrophys. J. Lett., 702, L37.

Mac Low M.-M. (1999) Astrophys. J., 524, 169.

Mac Low M.-M. and Klessen R. S. (2004) Reviews of Modern 
Physics, 76, 125.

Mac Low M.-M. et al. (1998) Physical Review Letters, 80, 2754.

Mamajek E. E. (2009) in: American Institute of Physics Conference Series, vol. 1158 of American Institute of Physics Conference Series, (edited by T. Usuda, M. Tamura, and M. Ishii), pp. 3-10.

Matzner C. D. and McKee C. F. (2000) Astrophys. J., 545, 364.

Maury A. J. et al. (2011) Astron. Astrophys., 535, A77.

McKee C. F. (1989) Astrophys. J., 345, 782.

McKee C. F. and Ostriker E. C. (2007) Annu. Rev. Astron. Astrophys., 45, 565.

McKee C. F. and Tan J. C. (2003) Astrophys. J., 585, 850.

Megeath S. T. et al. (2012) Astron. J., 144, 192.

Micic M. et al. (2012) Mon. Not. R. Astron. Soc., 421, 2531.

Molina F. Z. et al. (2012) Mon. Not. R. Astron. Soc., 423, 2680.

Muñoz D. J. et al. (2007) Astrophys. J., 668, 906.

Murray N. (2011) Astrophys. J., 729, 133.

Myers A. T. et al. (2013) Astrophys. J., 766, 97.

Nakamura F. and Li Z.-Y. (2008) Astrophys. J., 687, 354.

Nordlund Å. et al. (2013) in: Exploring the Formation and Evolution of Planetary Systems, vol. 299 of IAU Symposium, (edited by B. Matthews and J. Graham).

Nordlund Å. K. and Padoan P. (1999) in: Interstellar Turbulence, (edited by J. Franco and A. Carraminana), p. 218.

Offner S. S. R. et al. (2009) Astrophys. J., 703, 131.

Oliveira I. et al. (2009) Astrophys. J., 691, 672.

Onishi T. et al. (1998) Astrophys. J., 502, 296.

Ossenkopf V. and Mac Low M.-M. (2002) Astron. Astrophys., 390, 307.

Ostriker E. C. et al. (1999) Astrophys. J., 513, 259.

Ostriker E. C. et al. (2001) Astrophys. J., 546, 980.

Padoan P. (1995) Mon. Not. R. Astron. Soc., 277, 377.

Padoan P. and Nordlund A. (1997) ArXiv Astrophysics e-prints.

Padoan P. and Nordlund Å. (1999) Astrophys. J., 526, 279.

Padoan P. and Nordlund Å. (2011) Astrophys. J., 730, 40.

Padoan P. et al. (1997a) Astrophys. J., 474, 730.

Padoan P. et al. (1997b) Mon. Not. R. Astron. Soc., 288, 145.

Padoan P. et al. (1998) Astrophys. J., 504, 300.

Padoan P. et al. (1999) Astrophys. J., 525, 318.

Padoan P. et al. (2004) Physical Review Letters, 92, 19, 191102.

Padoan P. et al. (2005) Astrophys. J. Lett., 622, L61.

Padoan P. et al. (2006) Astrophys. J. Lett., 653, L125.

Padoan P. et al. (2009) Astrophys. J. Lett., 707, L153.

Padoan P. et al. (2012) Astrophys. J. Lett., 759, L27.

Passot T. and Vázquez-Semadeni E. (1998) Phys. Rev. E, 58, 4501.

Peters T. et al. (2010) Astrophys. J., 711, 1017.

Pilbratt G. L. et al. (2010) Astron. Astrophys., 518, L1.

Pineda J. L. et al. (2010) Astrophys. J., 721, 686.

Plume R. et al. (1997) Astrophys. J., 476, 730.

Price D. J. and Federrath C. (2010) Mon. Not. R. Astron. Soc., 406, 1659.

Price D. J. et al. (2011) Astrophys. J. Lett., 727, L21.

Price D. J. et al. (2012) Mon. Not. R. Astron. Soc., 423, L45.

Rebull L. M. et al. (2010) Astrophys. J. Suppl., 186, 259.

Renaud F. et al. (2012) Astrophys. J. Lett., 760, L16.

Ridge N. A. et al. (2006) Astron. J., 131, 2921.

Roman-Duval J. et al. (2010) Astrophys. J., 723, 492.

Roman-Duval J. et al. (2011) Astrophys. J., 740, 120.

Rosolowsky E. et al. (2010) Astrophys. J. Suppl., 188, 123.

Sadavoy S. I. et al. (2010) Astrophys. J., 710, 1247.

Sadavoy S. I. et al. (2012) Astron. Astrophys., 540, A10.

Sadavoy S. I. et al. (2013) Astrophys. J., 767, 126.
Scalo J. (1990) in: Physical Processes in Fragmentation and Star Formation, vol. 162 of Astrophysics and Space Science Library, (edited by R. Capuzzo-Dolcetta, C. Chiosi, and A. di Fazio), pp. 151-176.

Scalo J. and Elmegreen B. G. (2004) Annu. Rev. Astron. Astrophys., 42, 275.

Scalo J. et al. (1998) Astrophys. J., 504, 835.

Schleicher D. R. G. et al. (2013) New Journal of Physics, 15, 2 , 023017.

Schmidt M. (1959) Astrophys. J., 129, 243.

Schmidt M. (1963) Astrophys. J., 137, 758.

Schmidt W. et al. (2009) Astron. Astrophys., 494, 127.

Schmidt W. et al. (2013) Mon. Not. R. Astron. Soc., 431, 3196.

Schneider N. et al. (2012) Astron. Astrophys., 540, L11.

Schneider N. et al. (2013) Astrophys. J. Lett., 766, L17.

Schober J. et al. (2012) Phys. Rev. E, 85, 2, 026303.

Schuller F. et al. (2009) Astron. Astrophys., 504, 415.

Seifried D. et al. (2012) Mon. Not. R. Astron. Soc., 422, 347.

Shirley Y. L. et al. (2003) Astrophys. J. Suppl., 149, 375.

Shu F. H. et al. (1987) Annu. Rev. Astron. Astrophys., 25, 23.

Solomon P. M. et al. (1987) Astrophys. J., 319, 730.

Spezzi L. et al. (2008) Astrophys. J., 680, 1295.

Spezzi L. et al. (2010) Astron. Astrophys., 513, A38.

Stone J. M. et al. (1998) Astrophys. J. Lett., 508, L99.

Stutz A. M. et al. (2013) Astrophys. J., 767, 36.

Tasker E. J. and Bryan G. L. (2006) Astrophys. J., 641, 878.

Tassis K. et al. (2010) Mon. Not. R. Astron. Soc., 408, 1089.

Truelove J. K. et al. (1997) Astrophys. J. Lett., 489, L179.

Tsitali A. E. et al. (2010) Astrophys. J., 725, 2461.

Vazquez-Semadeni E. (1994) Astrophys. J., 423, 681.

Vázquez-Semadeni E. et al. (2003) Astrophys. J. Lett., 585, L131.

Vázquez-Semadeni E. et al. (2006) Astrophys. J., 643, 245.

Vutisalchavakul N. and Evans II N. J. (2013) Astrophys. J., 765, 129.

Wang P. et al. (2010) Astrophys. J., 709, 27.

Ward-Thompson D. et al. (2007a) Protostars and Planets V, pp. 33-46.

Ward-Thompson D. et al. (2007b) PASP, 119, 855.

Werner M. W. et al. (2004) Astrophys. J. Suppl., 154, 1.

Whitworth A. and Summers D. (1985) Mon. Not. R. Astron. Soc., 214,1 .

Williams J. P. and McKee C. F. (1997) Astrophys. J., 476, 166.

Williams J. P. et al. (2000) Protostars and Planets IV, p. 97.

Wu J. et al. (2005) Astrophys. J. Lett., 635, L173.

Wu J. et al. (2007) Astron. J., 133, 1560.

Wu J. et al. (2010) Astrophys. J. Suppl., 188, 313.

Young K. E. et al. (2006) Astrophys. J., 644, 326.

Yusef-Zadeh F. et al. (2009) Astrophys. J., 702, 178.

Zuckerman B. and Palmer P. (1974) Annu. Rev. Astron. Astrophys., 12, 279.

This 2-column preprint was prepared with the AAS LATEX macros v5.2. 